\title{
纳米碳材料在可穿戴柔性导电材料中的应用研究进展
}

\author{
夏凯伦寒木强张芗芗* \\ (清华大学化学系, 清华大学微纳米力学中心, 北京 100084)
}

\begin{abstract}
摘要: 可穿戴设备的兴起使得对柔性器件的需求日益提高, 柔性导电材料作为可穿戴器件的重要组成部分而 成为研究的热点。传统的电极材料主要是金属, 因金属材料本身不具有柔性, 一般通过降低金属层厚度以及 设计波纹结构等策略实现其在柔性器件中的应用, 其加工程序复杂, 成本较高。以碳纳米管和石墨烯为代表 的纳米碳材料兼具良好的柔性和优异的导电性, 且具有化学稳定、热稳定、光学透明性等优点, 在柔性导电 材料领域展现了极大的应用潜力。本文简要综述了近年来纳米碳材料在柔性导电材料领域的研究进展, 首先 介绍了碳纳米管基柔性导电材料, 分别包括基于碳纳米管水平阵列、碳纳米管垂直阵列、碳纳米管薄膜、碳 纳米管纤维的柔性导电材料; 继而介绍了石墨烯基柔性导电材料, 包括基于剥离法制备的石墨烯和化学气相 沉积法制备的石墨烯以及石墨烯纤维基柔性导电材料; 并简述了碳纳米管/石墨烯复合柔性导电材料; 最后 论述了纳米碳材料基柔性导电材料所面临的挑战并展望了其未来发展方向。
\end{abstract}

关键词: 碳纳米管; 石墨烯; 纳米碳材料; 柔性导电材料; 可穿戴器件

中图分类号: 0649

\section{Advances in Wearable and Flexible Conductors Based on Nanocarbon Materials}

\author{
XIA Kai-Lun JIAN Mu-Qiang ZHANG Ying-Ying* \\ (Center for Nano and Micro Mechanics, Department of Chemistry, Tsinghua University, Beijing 100084, P. R. China)
}

\begin{abstract}
With the rapid development of wearable devices, flexible conductive materials, which are one of the most important components of flexible electronics, have continued to attract increasing attention as important materials. Conventional electrodes mainly consist of rigid metallic materials, and consequently lack flexibility. Some of the strategies commonly used to make flexible metal electrodes include reducing the thickness of the electrode and designing electrodes with unique structural features. However, these techniques are generally complicated and expensive. Nanocarbon materials, especially carbon nanotubes and graphene, are highly flexible and exhibit excellent conductivity, superior thermal stability, good chemical stability, and high transmittance, making them good alternative materials for the preparation of flexible conductors. In this review, we have summarized recent advances towards the development of flexible conductors based on different types of nanocarbon materials, including carbon nanotubes arrays, carbon nanotubes films, carbon nanotubes fibers, graphene prepared using exfoliation or chemical vapor deposition techniques and graphene fibers. We have also provided a brief review of flexible conductive materials based on graphene/carbon nanotube composites, as well as a summary of the synthesis, fabrication and performances of these conductors. Finally, we have discussed the future challenges and possible research directions of flexible conductors based on nanocarbon materials.
\end{abstract}

Received: June 6, 2016; Revised: July 26, 2016; Published online: July 26, 2016.

${ }^{*}$ Corresponding author. Email: yingyingzhang@tsinghua.edu.cn; Tel: +86-10-62798503.

The project was supported by the National Natural Science Foundation of China (51422204, 51372132), Specialized Research Fund for the Doctoral Program of Higher Education, China (20120002120038), and National Key Basic Research Program of China (973) (2013CB228506).

国家自然科学基金(51422204, 51372132), 高等学校博士学科点专项科研基金(20120002120038)和国家重点基础研究发展规划项目(973) (2013CB228506)资助

(c) Editorial office of Acta Physico-Chimica Sinica 
Key Words: Carbon nanotube; Graphene; Nanocarbon material; Flexible conductive material; Wearable device

\section{1 引言}

随着社会对可穿戴设备的需求日益增长, 可 穿戴柔性器件的研究与开发被广泛关注。柔性导 电材料如同生物体的血管与神经, 是柔性器件中 不可或缺的重要组成部分。传统器件中的电极材 料以金属为主，由于金属不具有柔性，在拉伸过 程中易于发生断裂而失效, 无法满足柔性器件的 发展需求。目前, 柔性导电材料的设计思路主要 有三类：第一类是通过结构设计，将传统金属材 料设计为可拉伸结构, 例如美国西北大学的黄永 刚教授和伊利诺伊大学 Rogers 教授研究组, 将 金、铂等金属电极设计成分形结构 ${ }^{1}$, 从而用于柔 性传感器件并实现了在人体生理信号 ${ }^{2-5}$ 、温度 ${ }^{6}$ 、 皮肤含水量 78 和代谢产物等方面的检测 ${ }^{9-12}$ 。这类 结构具有优异的导电性, 但是加工过程通常需要 光刻、电子束沉积、反应离子刻蚀等微加工手 段, 操作复杂、成本高昂, 不宜于大规模应用。 第二类是以导电聚合物作为柔性电极材料, 常用 的导电聚合物有聚3-己基噻吩(P3HT) $)^{13}$ 、聚苯胺 (PANI)、聚吡咯(PPY)、聚3,4-乙撑二氧噻吩-聚苯 乙烯磺酸(PEDOT-PSS) 以及它们的衍生物 ${ }^{14-17}$ 。尽

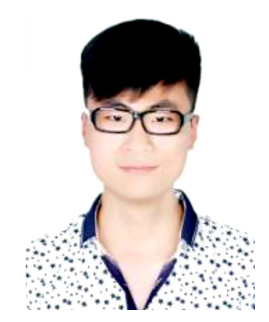

夏凯伦, 1994 年生。2015 年本科 毕业于北京化工大学材料科学与工 程专业, 2015 年至今为清华大学 化学系和清华大学微纳米力学与交 叉学科创新研究中心在读博士生。 主要研究方向为石墨烯的可控制备 及其在柔性器件中应用。
管导电聚合物兼具拉伸性和导电性，但是其在实 际应用中存在导电性较差等问题。第三类是将导 电纳米材料与柔性的基体材料通过一定方式结合来 制备柔性导电材料。此方法中, 导电纳米材料可选 取碳纳米管或石墨烯等纳米碳材料 ${ }^{18-20}$ 、金属纳米 线或金属纳米颗粒 ${ }^{21,22}$ 等, 其种类丰富, 选择灵活; 柔性基体材料可选取聚二甲基硅氧烷(PDMS)、聚 酰亚胺以及聚氨酯等。导电纳米材料可以直接沉 积在柔性材料表面或者与柔性基体材料混合形成 导电复合材料, 也可以将导电纳米材料设计成波 纹状、环状、网状以及弹簧状等可拉伸结构 $23-25$ 。 因此，将导电纳米材料与柔性基体材料复合具有 方法简单、原材料灵活多样、易于集成等特点, 是制备柔性导电材料的理想方法之一。

特别指出的是, 由于碳纳米管和石墨烯均具 有良好的柔性、出色的导电和导热性、优异的物 理与化学稳定性, 以及具有结构和性能易于调控 等特点, 近年来在柔性导电材料领域的应用得到 了广泛的关注和实质性的发展。本文综述了以碳 纳米管和石墨烯为代表的纳米碳材料在柔性导电 材料领域的发展现状与最新进展, 以期为纳米材 料以及柔性器件领域的相关研究人员提供参考。

\section{2 碳纳米管基柔性导电材料}

碳纳米管(carbon nanotube, CNT)具有极大的 长径比、优异的机械强度以及良好的导电导热能 力, 是一种理想的柔性导电材料。碳纳米管的制 备方法主要包括电弧放电法 ${ }^{25}$ 、激光烧蚀法 ${ }^{26}$ 和化 学气相沉积法 (chemical vapor deposition, CVD) 等 ${ }^{27}$ 。其中, CVD 法可控性强、成本低, 且易于实

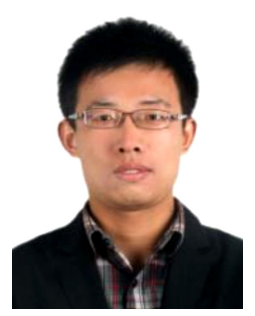

寒木强, 1989 年生。 2013 年本科 毕业于西北工业大学理学院化学工 程与工艺专业, 2013年至今为清华 大学化学系和清华大学微纳米力学 与交叉学科创新研究中心在读博士 生。主要研究方向为碳纳米管的可 控制备及其在柔性器件中的应用。

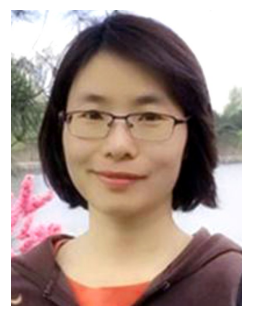

张莹荣, 1979年生。清华大学化学 系副教授，博士生导师。2007年于 北京大学获得博士学位, 2008年至 2011 年在美国洛斯阿拉莫斯国家实 验室从事博士后研究，2011年起任 职于清华大学。国家优秀青年基金 获得者。主要研究兴趣为纳米碳材料的可控制备 及其柔性可穿戴传感与器件。 
现碳纳米管的批量制备, 因此在基础科学研究和 工业生产应用中被广泛应用。CVD法制备的碳纳 米管有多种形貌，例如碳纳米管粉体、碳纳米管 水平阵列、碳纳米管垂直阵列、碳纳米管薄膜 等, 它们均可用于制备可穿戴柔性器件, 可以制 备为柔性导电材料或者柔性传感器件。本文重点关 注它们在柔性导电材料中的应用进展, 将分别概述 碳纳米管水平阵列基柔性导电材料、碳纳米管垂直 阵列(VACNT)基柔性导电材料、碳纳米管薄膜柔性 导电材料以及碳纳米管纤维柔性导电材料。

\section{1 碳纳米管阵列基柔性导电材料}

碳纳米管阵列是指阵列中的碳纳米管具有一 致取向的碳纳米管样品, 通常由基底承载, 其中 碳纳米管轴向与基底平行的样品称为碳纳米管水 平阵列, 碳纳米管轴向与基底垂直的样品称为碳 纳米管垂直阵列, 这两类阵列均可由 CVD 法直接 制备获得。下面分别介绍这两类材料在柔性导电 材料领域的应用。

\subsection{1 碳纳米管水平阵列基柔性导电材料}

碳纳米管水平阵列是指平行排列在基底表面 的碳纳米管, 这类碳纳米管因结构可控性强、定 向性好、缺陷含量少, 在电子器件领域中具有良 好的应用前景。2003 年, 美国杜克大学 Liu研究 组 ${ }^{28-30}$ 首次提出了气流导向化学气相沉积法, 通过
对基底和气体碳源前驱体采用不同的加热速率, 气体和基底之间的温度差会使碳纳米管沿着水平 方向浮动生长, 制备得到了水平取向的碳纳米管 阵列。此外还可以通过外加电场或者磁场的方法 来诱导碳纳米管取向 ${ }^{31-33}$ 。2007年, 美国伊利诺伊 大学的 Rogers 研究组 ${ }^{34}$ 首先开展了碳纳米管水平阵 列在柔性电子材料方面的研究, 他们在石英基底 表面生长出高密度碳纳米管水平阵列, 利用 PDMS 辅助转移法, 将碳纳米管水平阵列转移至硅片、 玻璃以及一些曲面结构, 包括柔性的塑料表面, 设计成柔性透明导体(图 $1(\mathrm{a}-\mathrm{d})$ ); 同时, 将单壁碳 纳米管水平阵列转移至预拉伸的 PDMS 基底表 面, 可以获得正弦波形式的屈服变形 ${ }^{35}$; 利用转移 至聚对苯二甲酸乙二醇酯(PET)基底表面的超高密 度碳纳米管阵列制备基本的逻辑器件 ${ }^{36}$, 器件迁移 率和跨导分别达到 $1000 \mathrm{~cm}^{2} \cdot \mathrm{V}^{-1} \cdot \mathrm{s}^{-1}$ 和 $3000 \mathrm{~S} \cdot$ $\mathrm{m}^{-1}$; 利用碳纳米管的网络结构, 在柔性基底(聚酰 亚胺)表面上制备了集成 100 个晶体管的全碳基的 逻辑电路, 其中晶体管的迁移率高达 $80 \mathrm{~cm}^{2} \cdot \mathrm{V}^{-1}$. $\mathrm{s}^{-1}$, 亚阈值斜率低至 $140 \mathrm{mV} \cdot \mathrm{dec}^{-1}$, 开关比达 $10^{5}$, 并展示了良好的机械柔性和性能一致性 ${ }^{37}$ 。

\section{1 .2 碳纳米管垂直阵列基柔性导电材料}

碳纳米管垂直阵列是由垂直于催化剂基底生 长并且相互之间近乎平行排列的碳纳米管宏观

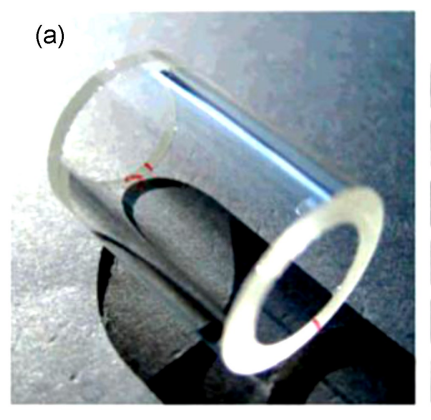

(c)

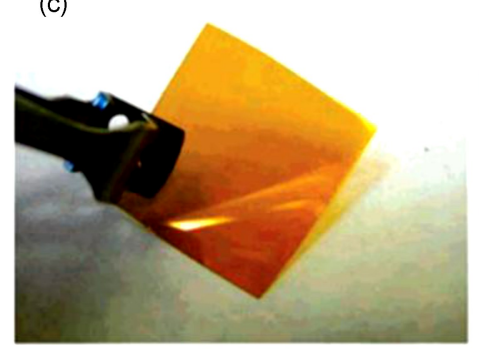

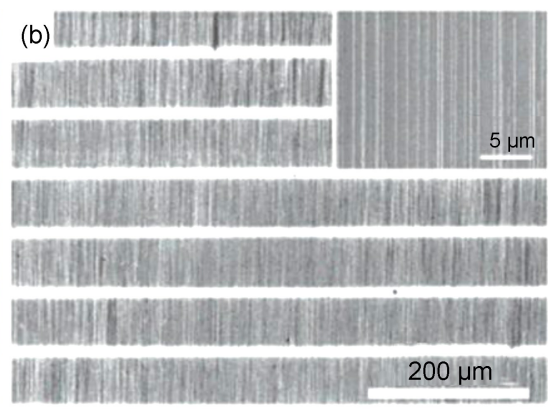

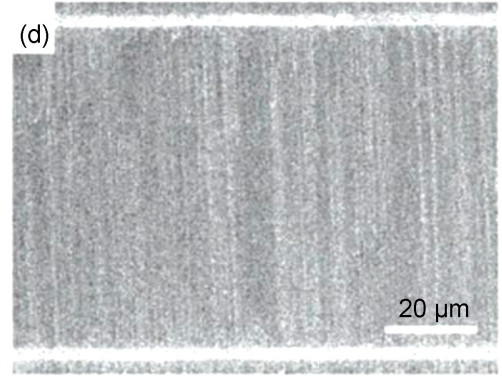

图 1 碳纳米管水平阵列基柔性导电材料 ${ }^{34}$

Fig.1 Horizontally aligned carbon nanotube arrays-based flexible conductor materials ${ }^{34}$

optical image (a) and SEM (b) of aligned arrays of single-walled carbon nanotube transferred from a quartz growth substrate to the surface of a glass cylinder; optical image (c) and SEM (d) of single-walled carbon nanotube transferred from a quartz growth substrate to the surface of a thin polyimide (Kapton) 
体。目前制备碳纳米管垂直阵列的主流方法是催 化剂薄膜辅助法, 使用预先沉积有高堆积密度纳 米级催化剂薄膜的基底来生长碳纳米管垂直阵 列, 可进行批量化生产。碳纳米管垂直阵列具有 碳纳米管高度取向性和优异的导电性等优势, 被 认为是理想的电极材料之一。

碳纳米管垂直阵列用于柔性可拉伸导体主要 有三种结构设计方法: 部分包埋垂直阵列、完全 包埋垂直阵列以及利用垂直阵列片层搭接平铺。 Tsai 等 ${ }^{38}$ 将图案化加工的碳纳米管垂直阵列直接转 移至透明的聚碳酸酯(PC)薄膜表面, 对垂直阵列施 加一定的温度(高于 $\mathrm{PC}$ 的玻璃化温度), 待冷却之 后, 碳纳米管阵列被薄膜部分包埋, 即可将特定 图案化的碳纳米管垂直阵列大面积转移至目标基 底。因结合牢固, 即使 PC 薄膜弯曲程度很大, 其 表面的垂直阵列仍然保持完好, 可用作压力传感 器。Kyoon 等 ${ }^{39}$ 采用滴落涂布法(drop-casting) 在碳 纳米管垂直阵列上方渗入聚氨酯(PU)溶液, 蒸发溶 剂后碳纳米管垂直阵列被 $\mathrm{PU}$ 完全包埋, 可直接从 硅片上剥离得到垂直阵列/PU 复合材料(图 2(a))。 以此为基础, 封装一层 PU 设计成三明治结构的可 拉伸导体, 拉伸应变可达到 1400\%。此外, 将垂 直阵列直接浸泡在 PDMS 前驱体溶液中, 形成 VACNT-PDMS 复合材料, 可用作压力传感器。当

施加压力时, 碳纳米管电极间的接触面积变大, 提供了更加密集的电流通道, 从而使电阻降低 ${ }^{40}$ 。 Shin 等 ${ }^{41}$ 利用一定图案结构的 PDMS 粘去图案之外 的垂直阵列, 再用 PDMS 溶液封装图案化的垂直 阵列, 转移到预拉伸的 PDMS 基底表面, 从而获 得波纹状结构的可拉伸复合材料(图 2(b))。波纹状 的结构赋予材料稳定的导电性和优异的拉伸性。 日本产业技术综合研究所的 Hata 研究组 ${ }^{42}$ 将垂直阵 列的片层转移至 PDMS 基底表面, 碳纳米管方向 与拉伸方向垂直, 并借助异丙醇溶液使碳纳米管 垂直阵列致密化, 将其用于应变传感器件 (图 2 (c))。碳纳米管垂直阵列在 PDMS 表面被拉伸时逐 渐形成小岛和间隙, 而间隙中会有碳纳米管束将 小岛连接在一起; 回复后, 表面的小岛连接成整 体。因此, 在拉伸和回复过程存在两个不同的应 变灵敏区, 主要来源于表面的岛和间隙的形成与 回复。依据这一性质设计成的应变传感器能够检 测 $0-280 \%$ 的应变, 而且 10000 次循环后性能仍然 良好, 具有耐久性、快速响应性和低蠕变性等优 点。碳纳米管应变传感器的灵敏系数和拉伸范围 普遍高于其他材料得到的应变传感器。该应变传 感器可集成在朋带、袜子以及手套等可穿戴配件 上进行人体运动的检测。

\section{2 碳纳米管薄膜基柔性导电材料}

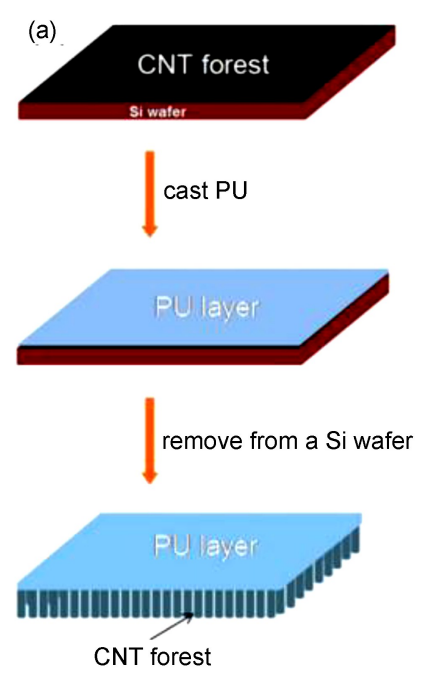

(b)
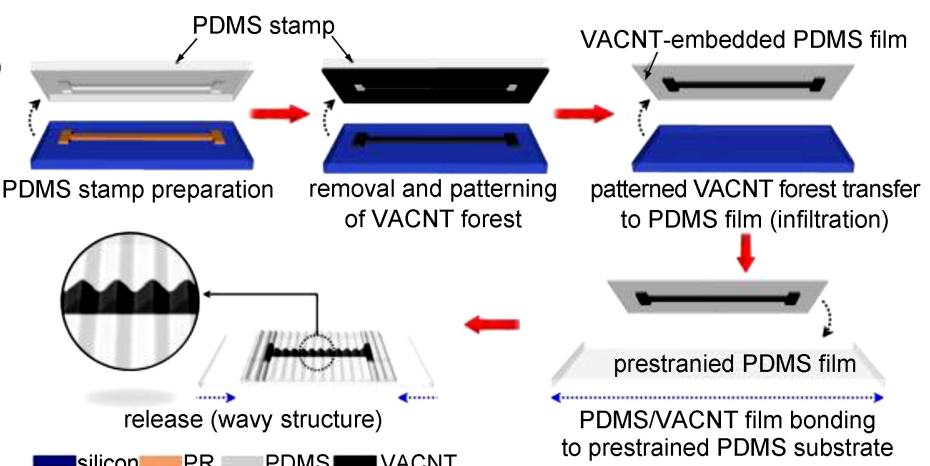

(c)

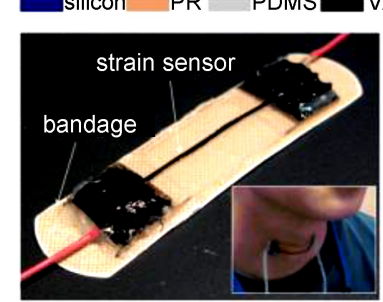

(d)

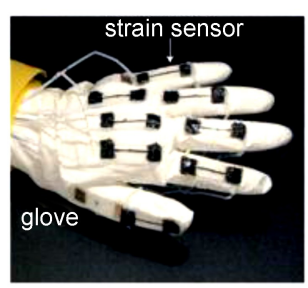

图 2 碳纳米管垂直阵列(VACNT)基柔性导电材料

Fig.2 Vertical array of carbon nanotube (VACNT)-based flexible conductor materials

(a) schematic diagram of the preparation method for the VACNT/polyurethane (PU) composite sheet ${ }^{39}$; (b) schematic diagram of the preparation process of polydimethylsiloxane (PDMS)/VACNT film based wavy configured stretchable conductors ${ }^{41}$; (c) photograph of bandage strain sensor;

(d) photograph of the sensors adhered to the glove $\mathrm{e}^{42}$ 
相比碳纳米管阵列, 碳纳米管薄膜的制备方 法与结构控制更为灵活多样，因此其柔性导电材 料的研究也更为广泛。碳纳米管薄膜的制备方法 可分为湿法和干法两类, 其中湿法是指将碳纳米 管经过溶液分散、纯化等处理制备为溶液, 然后 通过喷涂、棒涂、刮涂、滴落涂布以及真空抽滤 等方法制备得到碳纳米管薄膜; 干法是指不经过 溶液, 而是通过从碳纳米管垂直阵列直接拉膜、 卷压或者通过浮游催化气相沉积法(FCCVD)直接 收集薄膜等方式得到薄膜。下面将针对其在柔性 导电材料中的应用分别予以介绍。

\subsection{1 湿法制备的碳纳米管薄膜基柔性导电材料}

目前, 粉体碳纳米管已经实现工业化宏量制 备, 价格较低, 可以直接将碳纳米管分散在合适 的溶剂中，通过与聚合物前体直接混合或将溶液 采用一定方式涂布在聚合物基底表面等方法, 获 得高性能的薄膜复合材料。碳纳米管在溶剂中的 均匀分散是该类方法的关键。直接混合法得到的 碳纳米管/聚合物复合薄膜中碳纳米管含量少，且 碳纳米管长度短, 因此导电性一般; 涂布法, 例 如滴落涂布法、旋涂法、棒涂法、刮涂法、丝网 印刷法等，操作简单，适于大面积制备，因而具 有良好的应用前景, 但需要考虑碳纳米管薄膜与 聚合物结合力等问题 ${ }^{43-46}$ 。

Someya 等 ${ }^{45,46}$ 将碳纳米管混合在咪唑类离子液 体中，在喷射式粉碎技术辅助下均匀分散在高弹 性的复合共聚橡胶中形成 “巴基胶”，碳纳米管在 橡胶基体中形成良好的导电网络(图3(a-c))。由于 这种碳纳米管/像胶复合物的粘性较大，因此可以
直接印刷在柔性 PDMS 基底表面制成可拉伸导 体，拉伸性大于 $100 \%$ 且电导率大于 $100 \mathrm{~S} \cdot \mathrm{cm}^{-1}$ 。 在柔性可拉伸集成电路的制作中，巴基胶可作为 柔性导电材料将晶体管连接在一起, 在保证了导 电性的同时具有优异的可拉伸性 ${ }^{47}$ 。

Bao 等 ${ }^{48,49}$ 将碳纳米管分散在二甲基甲酰胺 (DMF)溶液中，采用喷涂技术把碳纳米管混合物喷 涂在预先用紫外/臭氧处理过的 PDMS 基底上。疏 水性的 PDMS 表面沉积碳纳米管时, 碳纳米管不 能均匀分散, 发生团聚; 而利用紫外/臭氧处理 后, PDMS 表面变成亲水性更有利于碳纳米管的均 匀分布。该复合物薄膜在拉伸时，由于碳纳米管 管间的接触会发生变化, 从而伴随着电阻的变 化, 由此得到了电阻式压力传感器 48 。这种传感器 可以测量 $0.1 \mathrm{~Pa}$ 以上的机械刺激但不能区分横向应 变和垂直压力。之后, 通过器件的结构设计, 该 课题组制备了可以区分并收集不同的机械刺激的 可拉伸器件, 可以同时测量横向应变产生的电容 变化和膜电阻变化且具有很高的压力灵敏度。除 此之外, 可以将不同的机械刺激转化成电压, 每 平方厘米电压和电流分别可以达到几十伏特和几 十微安, 从而实现自供电的功能。该器件可以附 着在皮肤上, 能够感知外界刺激, 并且人体运动 可为其持续提供能量，未来还可以进一步模拟人 体皮肤的功能和特性 ${ }^{49}$ 。

溶液法得到的碳纳米管普遍较短、缺陷较 多，且表面通常有表面活性剂残留，所得到的碳 纳米管薄膜的导电性并不理想，可通过加入金属 纳米颗粒、金属纳米线等方法提高该薄膜的导电
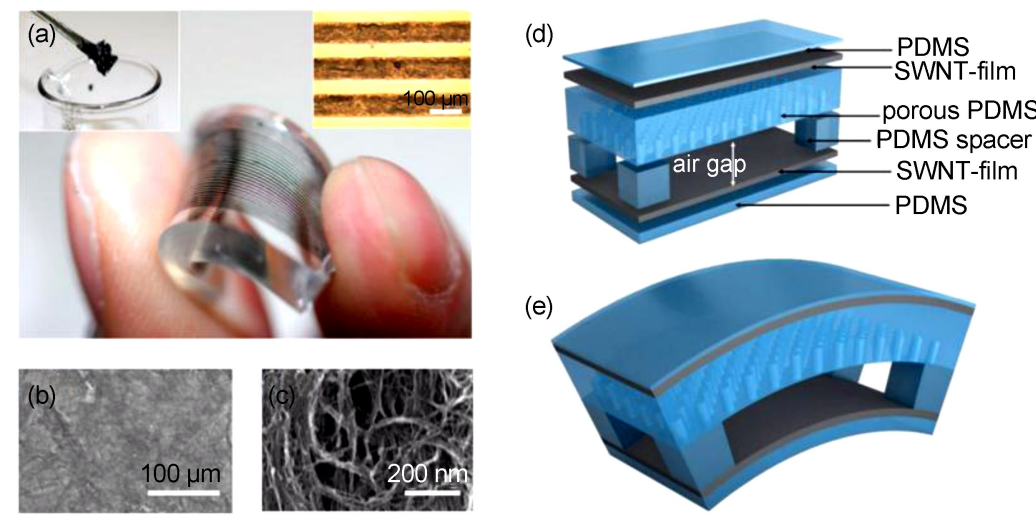

图 3 湿法制备碳纳米管(CNT)薄膜及其在柔性导电材料中的应用

Fig.3 Wet method for preparing carbon nanotube (CNT) film and the related application in flexible conductor materials (a) photograph of CNT/PDMS flexible conductor based on carbon nanotube solution ${ }^{45}$; (b) low resolution and (c) high resolution SEM images of carbon nanotube network ${ }^{46}$; $(d, e)$ schematic diagrams of energy-harvesting electronic skin device ${ }^{49}$ 
性。因此, 金属添加剂与碳纳米管复合柔性导体 近几年得到了广泛的研究。例如, Chun 等 ${ }^{50}$ 以碳 纳米管作为网络, 与 $\mathrm{Ag}$ 纳米颗粒复合制备了导电 薄膜。先在 $\mathrm{Ag}$ 纳米颗粒表面自组装苯基, 再将 $\mathrm{Ag}$ 纳米颗粒与碳纳米管进行混合, 在碳纳米管表面 接枝纳米级的 $\mathrm{Ag}$ 颗粒。然后加入离子液体经研磨 后得到凝胶, 与微米级片状 $\mathrm{Ag}$ 混合, 烘干得到 $\mathrm{Ag}$ /碳纳米管薄膜。当 $\mathrm{Ag}$ 微米片含量达到一定值 后, 薄膜电导率迅速增加, 但如果 $\mathrm{Ag}$ 微米片含量 超过 $8.6 \%$, 薄膜易碎且柔性降低。最后, 将橡胶 溶液与表面带有 $\mathrm{Ag}$ 颗粒的碳纳米管混合 ${ }^{51}$, 可制 备出弹性高导电性的粘胶剂, 增加 $\mathrm{Ag}$ 的含量可以 有效提高导电性并且橡胶本身与柔性基底不浸 润, 方便在柔性基底表面制备导电通道。此复合 材料在受力弯曲时比纯 $\mathrm{Ag}$ 或者纯碳纳米管均更稳 定。此外, Lee 等 ${ }^{52}$ 利用银纳米线与碳纳米管溶液 经过抽滤形成复合薄膜, 通过等离子焊接的方法 增强银纳米线之间的连接, 降低接触电阻以提高 导电性, 将该薄膜转移至 PET 基底上可得到柔性 导体。

\subsection{2 垂直阵列直接抽丝成膜制得的碳纳米管薄} 膜基柔性导电材料
由碳纳米管垂直阵列可以直接抽丝得到碳纳 米管薄膜并用于制备柔性导体。可直接抽丝成膜 的碳纳米管垂直阵列是碳纳米管垂直阵列中的一 类, 其中碳纳米管的取向和长度一致、直径分布 均匀, 从这类碳纳米管垂直阵列中可以直接抽出 连续的碳纳米管薄膜。2005 年, Baughman 研究 组 ${ }^{53}$ 在 Science 上报道了从碳纳米管垂直阵列中连 续地抽出碳纳米管透明薄膜, 碳纳米管之间由于 范德华力作用力而可以首尾相连得以连续地被抽 出来, 薄膜长度可达米级。实现这类碳纳米管薄 膜的连续化生产和工业化应用, 需制备出大尺寸 的可纺丝的碳纳米管垂直阵列。同一年, 清华大 学范守善研究组在 4 英寸的硅片上生长得到了碳纳 米管垂直阵列 ${ }^{54}$; 该研究组又在 8 英寸基底上制备 得到了碳纳米管垂直阵列, 实现了该类碳纳米管 垂直阵列以流水线的方式大量制备, 并结合传统 的干法纺丝技术 ${ }^{55-57}$ (图 4(a)), 得到了高度取向的 连续碳纳米管薄膜, 该薄膜具有良好的导电性(面 电阻为 $700-1500 \Omega \cdot \square^{-1}$ )、高柔韧性和优异的透光 性(单层膜在 $550 \mathrm{~nm}$ 可见光下透光率可达 $90 \%$ )。将 该薄膜转移至柔性基底表面，可用作柔性透明电 极、柔性发声器件、场发射器件、电/光驱动器件
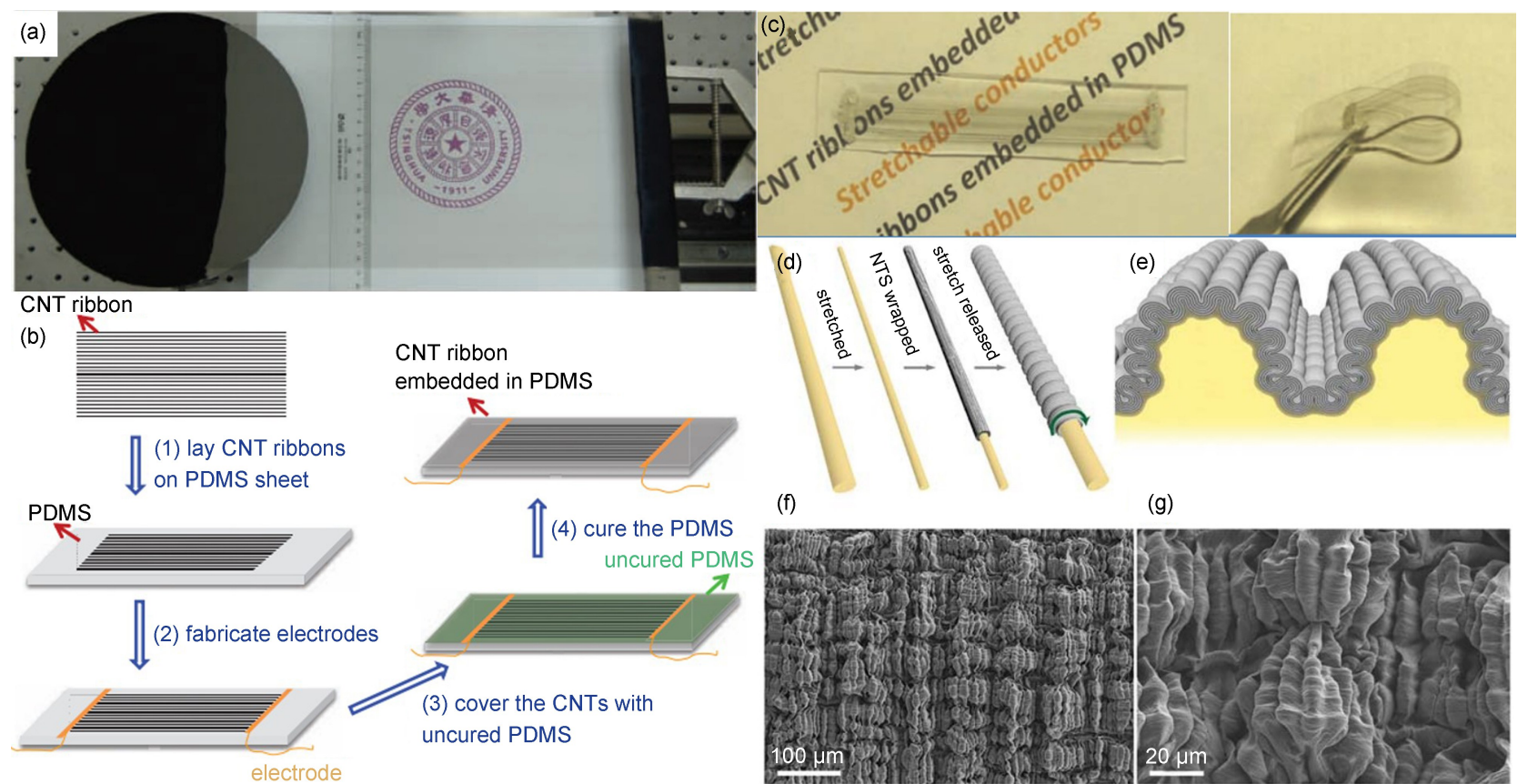

(f)

(g)
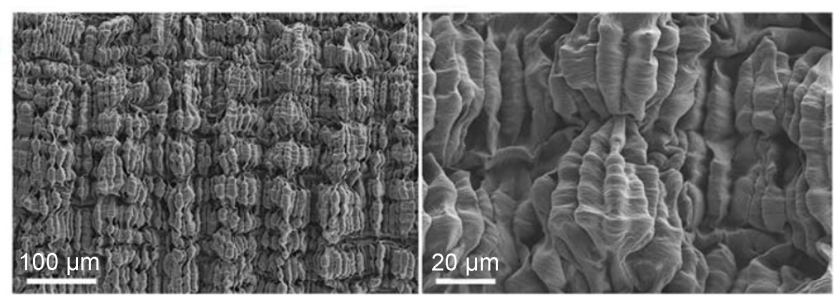

图4 碳纳米管垂直阵列直接抽丝成膜得到碳纳米管薄膜用于制备柔性可拉伸导体

Fig.4 Directly spun carbon nanotube film from vertical array of carbon nanotubes and the related application in flexible stretchable conductors

(a) optical image of carbon nanotube film from the VACNT ${ }^{56}$; (b) schematic diagram of embedding carbon nanotube film in PDMS; (c) optical image of CNT/PDMS film ${ }^{59}$; (d) schematic illustrate the fabrication of the CNT/rubber sheath-core fiber; (e) schematic illustrate the longitudinal section of the fiber; (f) low resolution and (g) high resolution SEM images showing long and short period buckles of the fiber under $100 \% \operatorname{strain}^{61}$ 
等。还可以采用卷对卷加工工艺批量化制备碳纳 米管/聚合物复合薄膜, 该薄膜柔韧性、拉伸性均 优于氧化铟锡(ITO)薄膜, 通过激光处理和沉积金 属等方法可提高其导电性, 是制备柔性手机触摸 屏等柔性屏幕的理想材料之一 ${ }^{58}$ 。

利用该种碳纳米管薄膜兼具导电性与柔性的 优点, 2010 年, Zhang 等 ${ }^{59}$ 首次将该类碳纳米管薄 膜转至 PDMS 中制备了透明可拉伸导体 (图 4(b, c))。对该碳纳米管/PDMS 薄膜进行拉伸试验, 在 第一次拉伸时, 因为拉伸过程中碳纳米管之间会 发生相对滑移, 其连接减弱甚至分离, 引起电阻 增加; 当释放应力时, 碳纳米管结构会发生重 排, 形成局部屈曲结构; 经历过几次拉伸-重排之 后, 碳纳米管薄膜的结构趋于稳定, 形成稳定而 均匀的屈曲结构; 当再次被拉伸时, 主要是屈曲 结构的伸展, 因此电阻不再发生变化, 可承受 $100 \%$ 应变而电阻保持不变, 是一种理想的柔性透 明导电材料。随后, $\mathrm{Zhu}$ 等 ${ }^{60}$ 尝试了将该类碳纳米 管薄膜转移至有预应变的 PDMS 上, 当应力释放 的时候, 碳纳米管形成波浪状屈曲结构, 因此可 用作柔性导电材料。2015 年, Baughman 研究组 ${ }^{61}$ 在预应变的橡胶纤维外侧包裹上数层碳纳米管薄 膜, 然后在外面覆盖上橡胶薄层, 再包裹数层碳 纳米管薄膜, 得到了高度可拉伸的核壳导电纤 维, 当轴向拉伸形变达到 $1000 \%$ 时, 其电阻仅变 化 5\%(图 4(d-g))。

\subsection{3 卷压法制备的碳纳米管薄膜基柔性导电 材料}

卷压法制备碳纳米管薄膜分为两步: 先用滚 动装置将碳纳米管垂直阵列推倒, 然后将其压实 以提高致密性, 而形成碳纳米管薄膜。2009年, Tawfick 等 ${ }^{62}$ 利用自制的机械装置推倒碳纳米管垂 直阵列, 并同时压实阵列, 从而获得紧密排列、 取向一致的碳纳米管薄膜, 电阻率仅为 $2 \mathrm{~m} \Omega$. $\mathrm{cm}^{-1}$ 。由于具有优异的导电性, 可以直接作为器件 连接导线或者转移至柔性基底表面(如 PDMS 和聚 酰亚胺), 用作应变传感器。Milne 研究组 ${ }^{63}$ 采用滚 动-碾压的方式用聚碳酸酯(PC)透明柔性薄膜转移 碳纳米管垂直阵列, 得到碳纳米管水平阵列薄膜/ PC复合材料。垂直阵列的高度越高, 获得的薄膜 导电性越好。相对于传统的 ITO而言, 这种复合薄 膜可以承受更大的形变且电学性能与 ITO 相当, 但 其透光率还有待进一步提高。Grigoropoulos 研究
组 ${ }^{64}$ 将聚合物与碳纳米管直接接触, 利用激光辅助 干法转移碳纳米管垂直阵列至聚合物基底。以 PC 为基底, 激光扫过的区域加热熔融 $\mathrm{PC}$, 使其包埋 垂直阵列并实现快速转移。通过控制激光扫过的 路径可以获取特定图案化的垂直阵列, 无须采用 光刻等技术。沿着不同的方向滚压垂直阵列, 可 以获得不同取向的碳纳米管薄膜。采用卷压法将 垂直阵列压实成膜操作简单, 薄膜的电阻可以通 过压实程度调节, 且易于转移到透明柔性聚合物 基底上用作柔性可拉伸导体。

\subsection{4 浮游催化化学气相沉积法直接制备的碳纳 米管薄膜基柔性导电材料}

采用浮游催化化学气相沉积法(FCCVD)直接 制备得到的碳纳米管薄膜也广泛用于制备柔性可 拉伸导体。中国科学院物理研究所的解思深院士 研究组 ${ }^{65}$ 采用FCCVD 法得到了厚度 $100 \mathrm{~nm}$ 的单壁 碳纳米管薄膜, 其透光率为 $70 \%$, 具有优异的导 电性和机械性能。2012年, 他们将 FCCVD法制备 的碳纳米管薄膜转移至 PDMS 表面, 制备了柔性 透明的导电材料 ${ }^{66}$ 。同年, 该研究组采用反复剥离 的方式来降低薄膜的厚度, 获得了透光率大于 $90 \%$ 的超薄碳纳米管薄膜, 并用于制备柔性透明超 级电容器 ${ }^{67}$ 。

\section{3 碳纳米管纤维基柔性导电材料}

碳纳米管纤维是由近似同轴排列的碳纳米管 组成, 具有极高的比模量、比强度和优异的电导 率以及热导率, 因此被广泛研究。目前碳纳米管 纤维的制备方法主要有湿纺和干纺两类, 其中干 纺包括从碳纳米管垂直阵列纺丝和通过 FCCVD法 纺丝两种方法。下面, 将分别介绍不同制备方法 所获得的碳纳米管纤维及其在柔性导电材料领域 的应用。

\subsection{1 湿法纺丝制备的碳纳米管纤维}

2000 年, 法国波尔多第一大学的 Poulin研究 组 ${ }^{68}$ 首次采用湿法纺丝法制备出碳纳米管纤维。图 5 (a) 是实验装置示意图。首先, 将单壁碳纳米管分 散在含有 $1.0 \%$ 的十二烷基硫酸钠(SDS) 的水溶液 中, SDS 会吸附在碳纳米管的表面防止碳纳米管 之间发生团聚, 从而可获得均匀分散的碳纳米管 溶液; 然后, 用注射器将碳纳米管溶液注入 $5 \%$ 的 聚乙烯醇(PVA)溶液中, PVA 会吸附在碳纳米管表 面并且替换掉部分的 SDS 分子, 从而可以实现连 续地抽出碳纳米管带, 图 5(b) 是得到的碳纳米管带 


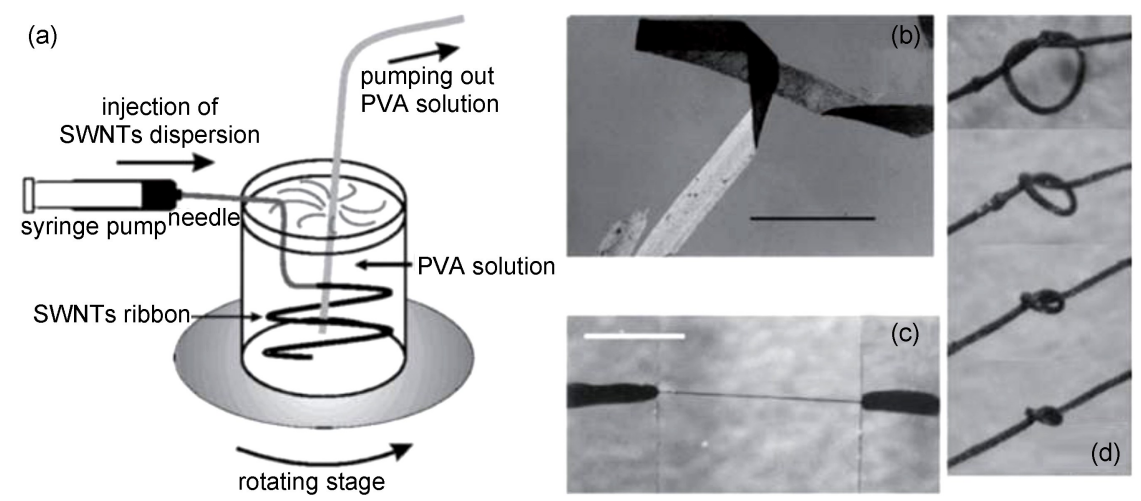

图 5 湿法纺丝制备碳纳米管纤维 ${ }^{68}$

Fig.5 Preparation of carbon nanotube fibers by wet spinning method ${ }^{68}$

(a) schematic of the experimental setup used to preparation of carbon nanotube fiber; (b) optical image of a single folded ribbon, scale bar: $1.5 \mathrm{~mm}$; (c) optical image of freestanding nanotube fiber between two glass substrates, scale bar: $1 \mathrm{~mm}$;

(d) optical image of fibers with knots, the fiber shown in the pictures has a radius of about $15 \mu \mathrm{m}$

的光学照片。之后, 将碳纳米管带洗涤干燥, 大 部分的分散剂和聚合物被去除, 碳纳米管带在毛 细管力作用下形成纤维(图 5(c))。X射线衍射结果 表明, 纤维的主要成分为单壁碳纳米管束、PVA 长链以及催化剂颗粒等 ${ }^{69}$ 。该纤维的性能和直径取 决于制备过程, 例如注射器直径, 注射速度以及 SDS、PVA 的浓度等。其中, 成纤的关键在于合适 的 SDS 浓度。该纤维具有优异的柔性(图 5(d)), 其 杨氏模量为 $40 \mathrm{GPa}$, 拉伸强度在 $300 \mathrm{MPa}$ 左右, 室温下的电导率约为 $10 \mathrm{~S} \cdot \mathrm{cm}^{-1}$ 。通过后处理, 例 如, 热拉伸过程, 可以增强纤维取向性和减小管 束间间距, 从而提高纤维的力学性能 ${ }^{70,71}$ 。

由于上述方法是在 PVA 溶液中进行的, 得到 的碳纳米管纤维中会含有大量的 PVA。PVA 的存 在可以提高纤维的成纤能力并改善其力学性能。 但是, PVA 是不导电的聚合物, 碳纳米管纤维中 高比例的 PVA 会降低纤维的导电性和导热性 ${ }^{69}$ 。因 此, 需要制备不含聚合物的碳纳米管纤维。2005 年, 莱斯大学的 Kozlov 等 ${ }^{72}$ 首次将单壁碳纳米管分 散在发烟硫酸中, 得到了浓度为 $8 \%$ 的单壁碳纳米 管均匀分散液。发烟硫酸对碳纳米管质子化作用 是碳纳米管均匀分散的关键, 单壁碳纳米管在硫 酸根离子的作用下有序排列。以水、5\%硫酸或者 乙二醚作为凝固浴, 可以得到直径 $50 \mu \mathrm{m}$, 长达 $30 \mathrm{~m}$ 的纯碳纳米管纤维。得到的纤维具有较好的 力学性能, 其拉伸强度为 $116 \mathrm{MPa}$, 杨氏模量为 $120 \mathrm{GPa}$ 。由于不含有聚合物, 纤维的电导率可以 达到 $500 \mathrm{~S} \cdot \mathrm{cm}^{-1}$ 。但是, 由于浓硫酸的质子化作 用, 会在纤维表面造成缺陷和孔洞, 降低纤维的力
学性能, 并且由于浓硫酸的存在, 对生产设备的要 求极为苛刻。为了解决这一问题, 一些研究组采用 其他的物质来代替发烟硫酸。例如, Steinmetz 等 ${ }^{73}$ 将 SDS 分散的单壁碳纳米管溶液注入甘油、乙醇 等小分子溶剂中, 硝酸铝盐作为凝固剂, 得到了 毫米级的碳纳米管纤维。Kozlov 等 ${ }^{74}$ 将单壁碳纳米 管分散在十二烷基苯磺酸锂(LDS)中, 在盐酸浴中 得到了碳纳米管纤维。当此纤维在氩气氛围下 $1000{ }^{\circ} \mathrm{C}$ 退火后, 可以得到纯碳纳米管纤维, 导电 率可以达到 $140 \mathrm{~S} \cdot \mathrm{cm}^{-1}$, 但是力学强度较低。

通过这类方法可以将无序的碳纳米管粉体材 料加工成连续的纤维, 易于工业化制备, 发展前 景较好。然而, 该类方法制备的碳纳米管纤维的 导电能力和力学性能还较差, 主要是因为在分散 碳纳米管时, 会引入表面活性剂或者强酸, 影响 碳纳米管的本征结构和性能, 从而降低纤维的导 电能力, 影响其力学性能。目前, 其力学性能还 低于商业碳纤维, 整体性能也有待进一步提高。 减少分散体系和分散过程对碳纳米管造成的缺陷 仍然是当前研究的重点。

\subsection{2 由碳纳米管垂直阵列干法纺丝制备的碳纳 米管纤维}

碳纳米管纤维也可以由碳纳米管垂直阵列通 过干法纺丝直接制备。碳纳米管成纤是一个自下 而上的过程, 与传统的碳纤维制备过程不同, 该 过程可以在室温下直接进行, 所得到的纤维具有 优异的电学和力学性能。

2002年, 清华大学范守善研究组 ${ }^{75}$ 首次在Nature 上报道了从碳纳米管垂直阵列中直接抽出长 30 
$\mathrm{cm}$, 直径 $200 \mu \mathrm{m}$ 的碳纳米管纤维, 发现其电学性 能突出。2004年, Baughman 研究组 ${ }^{76}$ 在 Science 上 报道了在碳纳米管垂直阵列抽丝过程中引入加捻 得到连续碳纳米管纤维的技术。所获得的纤维具 有高导电性和抗蠕变能力且强度超过 $460 \mathrm{MPa}$, 几 乎可以与防弹背心采用的纤维的强度相媲美, 经 过空气中 $450{ }^{\circ} \mathrm{C}$ 退火以及液氮中浸渍后, 仍然可 以保持较高强度。

这种方法制备的碳纳米管纤维的质量主要取 决于碳纳米管垂直阵列的质量。阵列的高度越 高, 碳纳米管纤维的力学性质越好, 这是因为超 长碳纳米管阵列形成的纤维端点缺陷少, 相邻碳 纳米管彼此接触紧密可以减少受力滑移 ${ }^{77}$ 。2007 年, Zhu 研究组 ${ }^{78}$ 从高度 $1 \mathrm{~mm}$ 的碳纳米管垂直阵 列中抽丝加捻制备了碳纳米管纤维, 其拉伸模量 和拉伸强度分别达到 263 和 $3.3 \mathrm{GPa}$ 。此外, 阵列 的密度、无定型碳的含量、直径以及管壁数均会 影响碳纳米管纤维的成纤性以及纤维质量。

这类碳纳米管纤维具有良好的导电性和柔 性, 可弯曲打结及拉伸, 因此可用于制备柔性导 体, 通过改变加入碳纳米管纤维的数量可以调节 柔性导体的电阻值和整体性能 ${ }^{24}$ 。

\subsection{3 由浮游催化化学气相沉积法直接纺丝制备 的碳纳米管纤维}

2004 年, 英国剑桥大学的 Windle 研究组 ${ }^{79}$ 在 Science 上首次报道了在浮游催化化学气相沉积法 制备碳纳米管过程中直接纺出碳纳米管纤维。他们 采用垂直 CVD炉, 以乙醇作为碳源, 二茂铁作为 催化剂, 将它们在载气的带动下自上而下地注入到 炉子的高温反应区以生长碳纳米管, 并且在低温区 将形成的碳纳米管气凝胶直接卷成碳纳米管纤维, 得到的纤维的纯度达到 $80 \%$ 并且具有较高的导电性 和断裂伸长率。之后该组对纺丝过程进一步研究, 使得到的碳纳米管纤维的强度、韧性等力学性能优 于许多商业高强度纤维 ${ }^{80}$ 。 $\mathrm{Li}$ 等 ${ }^{81}$ 采用该方法制备 了长达数千米的碳纳米管连续纤维, 且强度达到 1 $\mathrm{GPa}$ 以上, 证明了此法在批量生产上的可行性。 2015 年, Vilatela 等 ${ }^{82}$ 利用该方法通过优化条件制 备了取向高度一致的连续碳纳米管纤维, 通过降低 碳源供给量或者提高载气流量来降低气相中碳纳米 管的浓度, 从而降低纺丝过程中碳纳米管之间的缠 结, 可进行快速纺丝 $\left(50 \mathrm{~m} \cdot \mathrm{min}^{-1}\right)$ 。

碳纳米管纤维兼具柔性和导电性, 力学性能
优异, 因此可用作柔性导电材料, 纤维状的结构 赋予其可编织特性, 可以用于可穿戴设备中。目 前, 碳纳米管纤维在超级电容器电极 ${ }^{83-85}$ 、柔性太 阳能电池 ${ }^{86-88}$ 、锂离子电池电极 ${ }^{88,89}$ 等方面得到初步 应用探索。

\section{3 石墨烯基柔性导电材料}

自 2004 年英国曼彻斯特大学的 Geim 教授研究 组 ${ }^{90}$ 首次通过机械剥离法得到石墨烯以来, 石墨烯 一直是碳材料研究的前沿和热点。石墨烯是由 $s p^{2}$ 杂化的碳原子组成的蜂窝状结构, 单层石墨烯的 厚度仅为 $0.335 \mathrm{~nm}$, 其透光率高达 $97.7 \%$, 杨氏模 量达到 $1 \mathrm{TPa}$, 是目前已知强度最高、厚度最薄的 材料, 因其极佳的柔性和导电性, 被认为是制备 柔性导电材料的理想材料。目前, 制备石墨烯基 柔性导电材料按照石墨烯来源主要分为两大类: 一类是采用石墨烯溶液制备柔性电极材料, 所用 的石墨烯溶液为机械剥离的少层石墨烯分散液或 者化学剥离的氧化石墨烯 (GO)溶液; 另一类是通 过 CVD 得到高质量的单层或少层石墨烯, 然后转 移至柔性基底表面制成柔性导体。其中, GO 的制 备方法较为简单、成本低廉、可大规模生产, 后 续通过化学法还原、热还原、光还原等方法可得 到还原氧化石墨烯(rGO), 提高其导电性, 因此在 规模化生产应用方面存在优势。相比而言, CVD 法可以得到大片的石墨烯薄膜, 其结构和缺陷较 少, 性能优异, 然而, 该方法制备的成本较高, 并且其尺寸受限于 CVD 设备, 而且这样的石墨烯 薄膜需要被转移到柔性基底上以制备柔性导体, 在转移的过程中容易引入缺陷和杂质, 因此, 其 规模化应用还面临着诸多挑战。下面分别介绍这 两类方法制备的石墨烯在柔性导电材料中的应用。

\section{1 基于溶液法石墨烯的柔性导电材料}

氧化还原法和超声剥离法被广泛用来制备石 墨烯溶液, 其中, 氧化还原法制备过程简单, 原 材料易得, 产量高, 是宏量制备石墨烯最常用的 方法, 所得到的 GO 在水中具有良好的分散性, 可 以采用如真空抽滤、旋涂法、喷涂法等方式制备 成柔性薄膜材料。但是, 由于 $\mathrm{GO}$ 在制备过程中由 于强氧化剂和强酸的处理, 不可避免地引入大量 缺陷和含氧官能团, 导致其导电性较低, 通常需 要采用一定的手段将 $\mathrm{GO}$ 还原为 $\mathrm{rGO}$, 再将其应用 于制备柔性导体 ${ }^{91,92}$ 。 


\subsection{1 真空抽滤法}

2008 年, $E d a$ 等 $^{93}$ 首次报道了采用真空抽滤法 制备石墨烯柔性电极, 开创了这一领域的先河。 采用的 GO 分散液是浓度低至 $0.1-0.5 \mathrm{mg} \cdot \mathrm{L}^{-1}$ 的水 溶液。通过快速真空抽滤, GO 沉积到含有微孔的 纤维滤膜上(图 6(a,b))。由于 $\mathrm{GO}$ 膜的导电性较 差, Eda 等采用水合肼和低温退火的方式将 GO 还 原, 再转移至柔性 PET 表面制备成柔性透明导 体。真空抽滤过程中, 石墨烯片沉积到滤膜的表 面, 先将滤膜均匀覆盖, 然后再层层沉积。这种 方式得到的石墨烯膜均匀致密，而且石墨烯膜的 厚度可由石墨烯溶液的浓度和用量来调控。在此 基础上，后人又对该过程做了诸多改善，例如， Kim 等 ${ }^{94}$ 将制备的 $\mathrm{GO}$ 溶液先用肼进行预还原, 再 将溶液抽滤至阳极氧化铝 $(\mathrm{AAO})$ 薄膜上, $800^{\circ} \mathrm{C}$ 进 行高温还原, 得到石墨烯导体。最后用聚甲基丙 烯酸甲酯(PMMA)辅助转移到柔性基底表面得到柔 性石墨烯透明导体。该柔性透明导体的透光率可 达 $80 \%$, 电阻低至 $850 \Omega \cdot \square^{-1}$ 。

\section{1 .2 旋涂法}

石墨烯薄膜也可以通过旋涂法制备，旋涂法 对基底的洁净程度和石墨烯溶液的组成要求较高。 2008 年, Chen 研究组 ${ }^{95}$ 先用食人鱼溶液(Piranha solution, 体积比为 $7: 3$ 的 $\mathrm{H}_{2} \mathrm{SO}_{4}: \mathrm{H}_{2} \mathrm{O}_{2}$ 混合溶液) 将石英片表面处理干净, 再在表面沉积一层三乙 基氧硅烷(APTES), 使表面接触角为 $67^{\circ}$, 得到亲 水性表面; 然后将 $\mathrm{GO}$ 水溶液旋涂在基底表面, 得
到平整的 $\mathrm{GO}$ 膜; 薄膜干燥之后用肼和低温退火结 合对 $\mathrm{GO}$ 进行还原, 得到导电的石墨烯膜。旋涂法 制备石墨烯薄膜需要严格控制 GO 溶液和转速, 转速 增大可以加快溶剂蒸发, 减小膜的厚度。Robinson 等 ${ }^{96}$ 在 $\mathrm{GO}$ 水溶液中加入一定量的乙醇, 旋涂在硅 片基底表面之后, 用氮气枪加快溶剂挥发, 干燥 之后在基底表面得到了纳米级厚度的石墨烯导电 膜(图 6(c)); 用肼还原之后将样品放置于氢氧化钠 溶液中刻蚀 $\mathrm{SiO}_{2}$ 层, 石墨烯膜会浮在液体表面, 再用柔性基底捞取, 得到柔性导电电极。Zheng 等 ${ }^{97}$ 先用 Hummers 法制备 GO 悬浮液, 用表面活性 剂十二烷基苯磺酸钠(SDBS)来处理 GO, 有利于得 到大面积的石墨烯片, 再将 SDBS-GO 混合溶液用 肼在 $100{ }^{\circ} \mathrm{C}$ 下还原 $24 \mathrm{~h}$ 。然后加入导电高分子 (PEDOT:PSS), 混合均匀, 在 PET 基底上旋涂, 蒸 发掉溶剂得到柔性石墨烯导体。这种常温下制备 的石墨烯柔性导电膜具有良好的导电性和优异的 耐弯折性能。

\section{1 .3 自组装法}

石墨烯薄膜也可以通过自组装法进行制备。 例如, Zheng 等 ${ }^{98}$ 利用 Langmuir-Blodgett (LB) 自组 装方法制备了含有超大石墨烯片的柔性导电膜, 将水/乙醇溶液按照体积比 $1: 5$ 混合填充到 LB 槽 中，再把 $\mathrm{GO}$ 分散液缓慢的注射到液体表面，表面 张力促使石墨烯片充分分散从而得到石墨烯膜。 在制备石墨烯导电膜过程中, GO 形成连续的层层 紧密堆积的薄膜, 烘干之后经过还原得到多层石

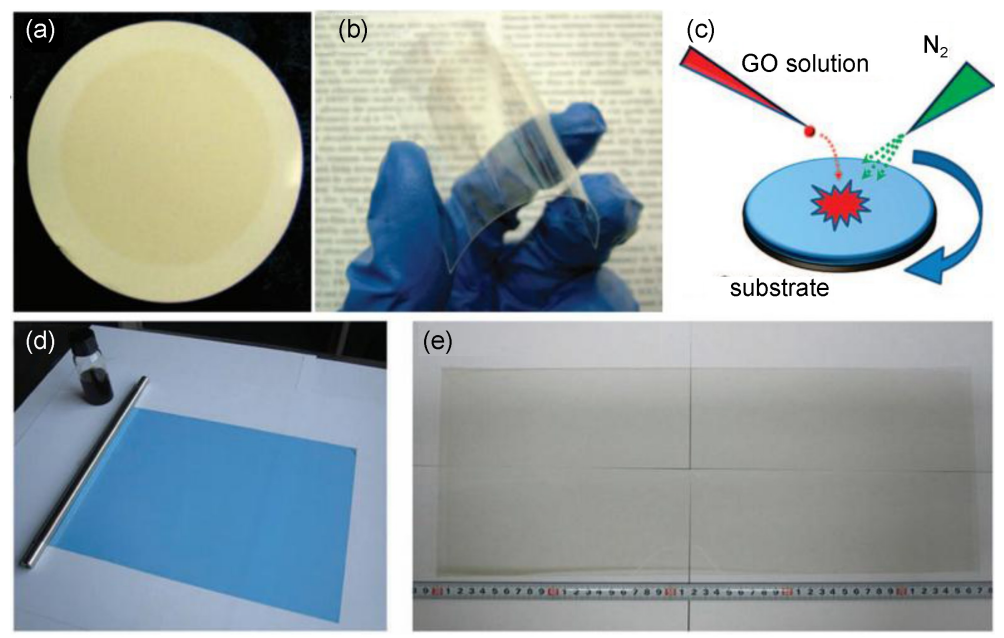

图 6 利用石墨烯溶液制备柔性导电薄膜

Fig.6 Flexible conductive film prepared from graphene solution

photographs of GO thin film on (a) filtration membrane and (b) plastic substrates ${ }^{93}$; (c) cartoon of spin coating process assisted by nitrogen flow ${ }^{96}$; (d) rod-coating experimental setup; (e) photograph of large-scale GO film coated on PET substrate ${ }^{106}$ 
墨烯薄膜。此外, Cheng 研究组 ${ }^{99,100}$ 利用气/液界面 自组装的方式制备了可自支撑的 $\mathrm{GO}$ 薄膜, 这种方 法得到的石墨烯薄膜厚度均匀, 且厚度和尺寸可 控, 能够方便地转移至各种基底表面。这种气液 界面自组装过程中, $\mathrm{GO}$ 单层片因含氧官能团之间 具有强烈的静电排斥作用, 层与层之间以及边缘 与边缘之间的排斥作用, 可以有效地避免石墨烯 片团聚，从而促进了均匀石墨烯薄膜的形成 ${ }^{101}$ 。用 石墨烯溶液也可以通过 LB 膜技术来制备大面积薄 膜, 例如, Dai 研究组 ${ }^{102}$ 用超声剥离方式制备出高 质量的单层石墨烯碎片, 分散在有机溶剂中, 采 用 LB 层层自组装方式得到大面积柔性石墨烯导电 膜, 面电阻为 $8 \mathrm{k} \Omega \cdot \square^{-1}$ 的石墨烯导电膜的透光率 可达 $83 \%$ 。

\section{1 .4 其他制备方法}

Cheng 等 ${ }^{103}$ 采用电泳沉积方式制备了高密度、 厚度均匀、边缘结构平整的石墨烯导电薄膜。他 们先用超声法制备了大尺寸石墨烯的异丙醇分散 液, 向溶液中加入一定量的硝酸镁溶液使石墨烯 表面带上正电荷, 然后通过电泳沉积的方式, 形 成石墨烯薄膜。该石墨烯薄膜的厚度可以通过调 节硝酸镁的加入量、电泳采用电压以及沉积时间 来调控。Lee等 ${ }^{104}$ 将 GO 溶液浇筑在干净的玻璃培 养皿内, 让溶液自然蒸发再真空烘干，得到了均 匀密实的 GO 薄膜, 再用氢碘酸 $(\mathrm{HI})$ 蒸汽还原后转 移至 PET 表面得到了柔性透明导电材料。Zhi 研究 组 ${ }^{105}$ 将 GO 溶液与氯化钯溶液混合, 用线棒涂膜方 式将 $\mathrm{GO} / \mathrm{Pd}$ 混合溶液均匀涂敷在 PET 基底表面, 然后在室温下采用高压蒸汽加压方式用氢气还原 得到了大面积柔性透明石墨烯导电膜(图 6(d, e))。 Chen 等 ${ }^{106}$ 将 $\mathrm{GO}$ 溶液滴涂到基底表面, 并用肼还原 方式得到 $10 \mathrm{~nm}$ 厚的柔性石墨烯导电膜。从石墨烯 水溶液得到石墨烯薄膜的研究工作还有很多, 发 展出许多成熟的方法, 极大地促进了石墨烯基柔 性导电材料的发展。

在溶液法制备石墨烯导电薄膜的基础上, 研 究者在改进导电性方面做了很多研究。其中, 金 属纳米线与石墨烯复合是提高导电性的重要手段 之一 ${ }^{107,108}$, 该过程可以大幅度提高导电性而不引起 透光率的明显降低。例如, Alam 等 ${ }^{109}$ 研究了金属 纳米线与多晶石墨烯在复合薄膜中的协同机制, 金属纳米线在石墨烯表面形成的网络有利于增加 石墨烯晶界之间连接的桥梁, 在显著提高电导率
的同时, 对透光率影响很小。Jung 等 ${ }^{110}$ 发现金属纳 米线与石墨烯复合材料的电导率提高不仅来源于 金属纳米线的掺杂，而且石墨烯自身的电导率在 金属纳米线的存在下会提高 $30 \%$, 这就意味着, 金属纳米线与石墨烯之间的接触对复合薄膜的导 电性能影响很大, 为了优化复合材料的导电性 能, 需要改善石墨烯与金属线以及金属线之间的 接触。Xu等 ${ }^{111}$ 在 $\mathrm{GO}$ 溶液中加入银纳米线, 通过湿 法纺丝得到了银纳米线掺杂石墨烯纤维, 再经过 化学还原提高导电性, 其中银纳米线沿着轴向排 列更均匀, 纤维的电导率可达到 $960 \mathrm{~S} \cdot \mathrm{cm}^{-1}$ 。Tour 等 ${ }^{112}$ 制备了金属网格与石墨烯的复合透明导电薄 膜, 薄膜电阻在透光率 $80 \%$ 下为 $3 \Omega \cdot \square^{-1}$, 透光率 $90 \%$ 时其电阻也仅为 $20 \Omega \cdot \square^{-1}$ 。

\section{2 基于 CVD 石墨烯的柔性导电材料}

CVD 法可以制备出大面积高质量的石墨烯 膜, 相比于溶液法而言, CVD生长的石墨烯结晶 性更好, 导电性更优 ${ }^{113}$ 。由于 CVD生长石墨烯采 用的基底一般是金属铜或者镍，这类金属基底柔 性较差且不透明, 因此需要将石墨烯转移到柔性 基底上以用作柔性导电材料。2009年，Kim 等 ${ }^{23}$ 在 Nature 上首次报道了利用 CVD 法生长的石墨烯制 备柔性可拉伸透明导体。研究者在硅片表面沉积 了一层图案化的金属镍层, 在 $1000{ }^{\circ} \mathrm{C}$ 下, CVD 法生长出少层石墨烯薄膜, 然后在表面封装一层 PDMS, 固化之后将镍层刻蚀掉, 再将石墨烯层转 印在目标基底上; 或者, 直接将镍层刻蚀掉, 石 墨烯层漂浮在液体表面, 然后用目标基底捞取悬 浮的石墨烯层, 从而得到柔性透明导电电极, 其 透光率达到 $80 \%$, 电阻仅为 $280 \Omega \cdot \square^{-1}$, 并且具有 优异的光电性能(图 7 $(\mathrm{a}-\mathrm{c})$ )。同年, Ruoff 等 ${ }^{114}$ 在 铜箔表面生长得到大尺寸单层、双层和少层石墨 烯膜, 并在 PMMA 辅助下大面积完整地转移至 PET 表面, 得到了低电阻高透明性的柔性导电电极 (图 7(d))。Bae 等 ${ }^{115}$ 采用卷对卷方式, 将铜箔上生 长的大面积单层石墨烯转移至 PET 表面, 得到了 尺寸达 30 英寸的柔性透明导电电极, 薄膜电阻低 至 $125 \Omega \cdot \square^{-1}$, 透光率高达 $97.4 \%$, 表现出半整数 量子霍尔效应。进一步采用层层堆叠的方式得到 了含有四层石墨烯的柔性透明导电电极, 其透光 率达到 $90 \%$, 电阻仅为 $30 \Omega \cdot \square^{-1}$ 。他们进一步用 这种导电薄膜制成了电子触摸屏, 证明了石墨烯 导电电极替代传统商业电极的可能性。 

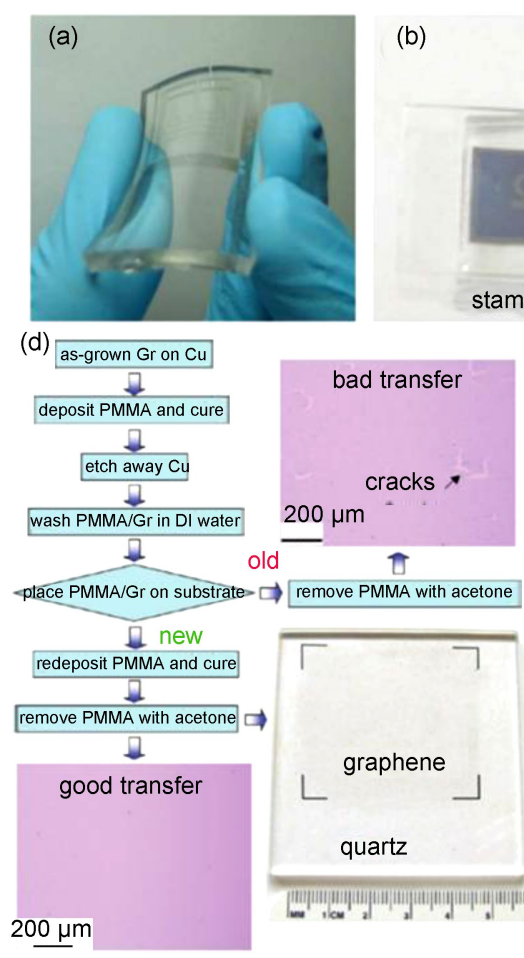

(b)

(c)

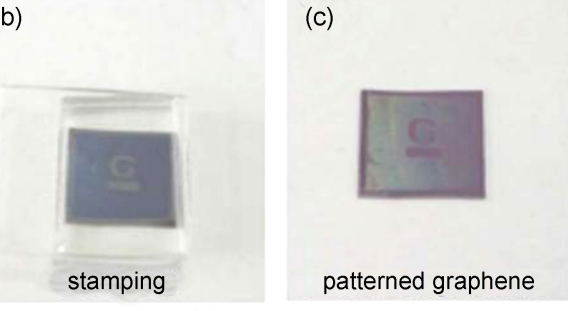

(e)

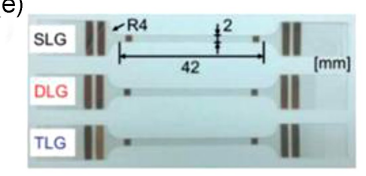

electrical resistance

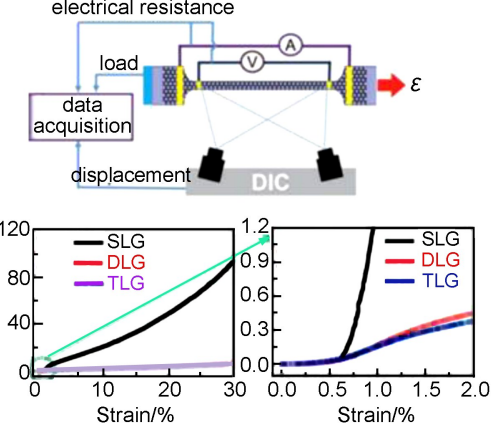

图 7 基于 CVD生长的石墨烯薄膜制备柔性可拉伸导电薄膜

Fig.7 Flexible conductor based on graphene film synthesized by CVD

(a) optical image of graphene film on flexible PDMS substrate; (b, c) PDMS stamp makes conformal contact with a silicon dioxide substrate ${ }^{23}$;

(d) transfer process of graphene film and the optical image on $\mathrm{SiO}_{2} / \mathrm{Si}\left(285 \mathrm{~nm} \mathrm{SiO}{ }_{2}\right.$ layer $)$ and quartz substrates ${ }^{115}$;

(e) photograph of single- and multi-layer graphene flexible conductor and electromechanical tensile testing ${ }^{117}$

在多层石墨烯薄膜中, 石墨烯层之间可以发 生滑移，从而提供一定的可拉伸性。Won 等 ${ }^{116}$ 将石 墨烯薄膜转移至 PET 基底表面, 研究表明, 单层 石墨烯薄膜在 PET 上当应变达到 $4.5 \%$ 时就出现了 缺陷和破坏，而双层石墨烯薄膜的可拉伸性达 $36.2 \%$ (图 7(e))。此外, 为了增加石墨烯薄膜的可 延展性, Dai 等 ${ }^{117}$ 先用铌子夹持铜箔得到波纹状铜 箔结构, 再 CVD生长石墨烯, 然后在表面旋涂 PDMS, 固化之后刻蚀掉铜箔, 得到了波纹状可拉 伸石墨烯电极。

基于 CVD法石墨烯制备柔性导电材料面临的 主要挑战是石墨烯的转移问题。该转移过程往往 涉及聚合物或者刻蚀性化学溶液, 这些物质的残 余会影响其性能, 例如, 残留的有机聚合物将增 大电极材料的电阻并降低电子迁移率, 这些问题 需要通过探索新的转移过程进行解决。

\section{3 石墨烯纤维基柔性导电材料}

宏观的石墨烯纤维可由微观石墨烯片层通过 一定纺丝方法得到, 所得到的石墨烯纤维不仅可 以保持石墨烯优异的导电性、导热性等性能, 而 且具有优良的柔性和力学性能, 从而可以作为性
能优异的柔性导电材料。下面，将分别介绍石墨 烯纤维的不同的制备方法，在此基础上，介绍其 在柔性导电材料领域中的应用 118,119 。

\subsection{1 石墨烯纤维的制备}

石墨烯纤维的制备主要参考了传统的纺丝手 段 ${ }^{120}$, 主要方法分为湿法纺丝、干法纺丝以及干喷 湿纺等。此外, 石墨烯纤维也可通过电泳法以及 石墨烯薄膜提拉成纤维等方法进行制备。

\subsubsection{1 湿法纺丝法制备石墨烯纤维}

湿法纺丝制备石墨烯纤维需要用到稳定分散 的 GO 溶液。在纺丝过程中, 用注射器将纺丝液注 入凝固浴, 得到凝胶状的 $\mathrm{GO}$ 纤维。为了保证 $\mathrm{GO}$ 纤维的均匀性和连续性, 一般需要在纺丝过程中 旋转凝固浴或转动收集单元从而以一定速度牵引 纤维(图 8(a,b))。相比于旋转凝固浴 ${ }^{120}$, 转动收集 单元可以提供稳定的牵引力和转动速度, 能更好 的保证纤维的性能。凝胶状 GO 纤维经过干燥后得 到力学性能优异的 $\mathrm{GO}$ 纤维, 还原后即可得到导电 性良好的 rGO纤维。

湿法纺丝制备 $\mathrm{GO}$ 纤维的本质是液晶相湿法纺 丝, $\mathrm{GO}$ 片层的高纵横比以及 $\mathrm{GO}$ 层间强作用力对 


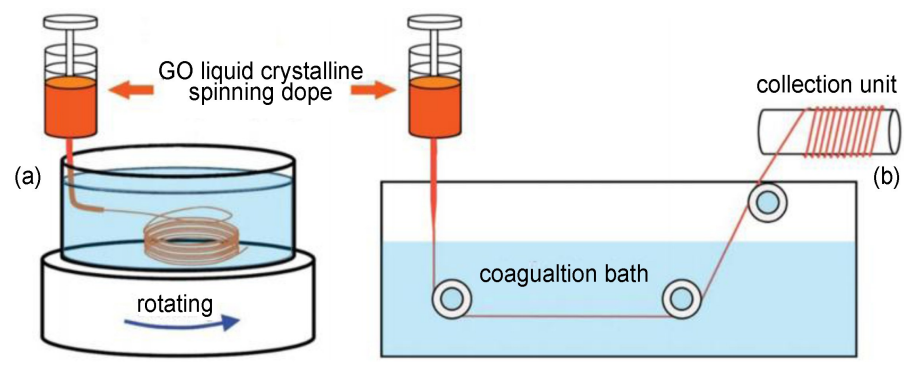

图 8 湿法纺丝制备石墨烯纤维装置

Fig.8 Wet-spinning setup used for graphene fiber

schematic of (a) a rotating coagulation bath and (b) a rotating collection unit

于自组装成连续的高性能的石墨烯纤维至关重 要。湿法纺丝制备石墨烯纤维的关键是控制 $\mathrm{GO}$ 纺 丝液和凝固浴。

$\mathrm{GO}$ 纺丝液的可纺丝性是由 GO 片层的尺寸和 溶液的浓度决定的。对于大尺寸的 GO纺丝液，只 有当纺丝液中的 $\mathrm{GO}$ 完全为液晶相并大于临界浓度 时才具备可纺丝性, 否则, 得到的产物会是一些 不连续的纤维或者团聚物。Jalili 等 ${ }^{121}$ 发现, 当 GO 片层的公称直径 $(D)$ 为 $37 \mu \mathrm{m}$ 时, 全液晶相的 $\mathrm{GO}$ 纺 丝液的浓度只有大于临界浓度 $\left(0.75 \mathrm{mg} \cdot \mathrm{mL}^{-1}\right)$ 时才 能保证连续的纺丝。Xu等 ${ }^{122}$ 报道, 当 $\mathrm{GO}$ 片层的 $D=25 \mu \mathrm{m}$ 时, 临界浓度为 $4 \mathrm{mg} \cdot \mathrm{mL}^{-1} ; D=0.91$ $\mu \mathrm{m}$ 时, 临界浓度为 $81 \mathrm{mg} \cdot \mathrm{mL}^{-1}$ 。 $\mathrm{GO}$ 的液晶相取 向结构在凝胶状和干燥的纤维中都能保持, 即使 对 GO 片层进行掺杂也不会影响其取向性和纺丝 性, 由此可以制备聚合物或者金属纳米粒子掺杂 的石墨烯复合纤维 ${ }^{111,123-125}$ 。

除了 GO 纺丝液, 凝固浴在石墨烯纤维的制备 过程中也起到重要的作用。GO纺丝液是由带负电 的 GO 片层稳定分散在水中得到的, 而当制备纤维 时, 需要打破这种稳定的状态, 让 $\mathrm{GO}$ 沉降形成凝 胶状, 这一过程通常使用凝固浴来实现。GO表面 的含氧官能团使其可以稳定分散在极性或者可与 GO 形成氢键作用的溶液中, 当其在非极性的溶液 中就会产生沉降。Xiang 等 ${ }^{126}$ 使用乙酸乙酯凝固 浴, 制备了高性能的石墨烯纤维。当带负电的 GO 遇到相反电荷的离子的破坏作用时也会发生沉 降, 例如, 具有两亲性、带相反电荷的聚合物或 离子、二价阳离子等可以作为凝固剂促使 $\mathrm{GO}$ 发生 沉降 ${ }^{121,127-129}$ 。

凝胶状 GO 纤维体积的 $67 \%-90 \%$ 是凝固剂和 残留的溶剂, 干燥过程中溶剂挥发, GO 纤维的直 径会降至原来凝胶状纤维直径的 $1 / 10$ 至 $1 / 3$ 。在纤
维纺丝过程中, $\mathrm{GO}$ 片层会沿着纤维轴向取向, GO 片层之间存在着溶剂, 在干燥过程中, 随着溶 剂的蒸发, GO片层会发生折叠及弯曲, 干燥后的 纤维表面有沿着纤维轴向的大量褶皱, 促进孔隙 的生成和比表面积的增加。此外, 通过调控湿法 纺丝方式, 可以得到不同结构的石墨烯纤维。Kou 等 ${ }^{130}$ 采用同轴的两个喷头, $\mathrm{GO}$ 采用内部喷头, 羧 甲基纤维素钠 $(\mathrm{CMC})$ 采用外部喷头, 一步法合成了 核壳结构的石墨烯纤维。 $\mathrm{CMC}$ 是离子导体但是电 绝缘体, 从而使纤维在编织成的织物中不会发生 短路。Zhao 等 ${ }^{131}$ 采用类似的装置, 外部喷头填充 了 $\mathrm{GO}$, 内部喷头填充了流动的凝固剂, 制备得到 了连续的中空结构的石墨烯纤维。

\subsubsection{2 干法纺丝法制备石墨烯纤维}

干法纺丝也是以 $\mathrm{GO}$ 的稳定分散液作为纺丝 液, 和湿法纺丝相比, 干法纺丝不需要使用凝固 浴, 而是将纺丝液注入并封闭在一维管状模具 中, 采用加热或高温化学还原方式使溶剂挥发, $\mathrm{GO}$ 沉降堆叠成纤维。 $\mathrm{Yu}$ 等 ${ }^{132}$ 将 $\mathrm{GO}$ 分散液注入到 一维管内, $220-230{ }^{\circ} \mathrm{C}$ 下加热得到石墨烯纤维。 该纤维的长度可达数米, 拉伸强度可达 $180 \mathrm{MPa}$ 。 这种方式得到的纤维实质上是 $\mathrm{rGO}$ 纤维, 因为高 温促使 GO 发生了还原。Acik 等 ${ }^{133}$ 研究发现 GO 在 $180{ }^{\circ} \mathrm{C}$ 下 $27 \%$ 的含氧官能团会被去除掉, 大多数的 羟基、环氧基团以及羧基在 $200{ }^{\circ} \mathrm{C}$ 下也会发生解 离。干法纺丝得到的石墨烯纤维省去了还原过 程, 具有较好的电导率 $\left(10 \mathrm{~S} \cdot \mathrm{cm}^{-1}\right)^{134}$ 。 $\mathrm{Li}$ 等 ${ }^{135}$ 将 $\mathrm{GO}$ 和维他命 $\mathrm{C}$ 溶液共同注入聚丙烯 $(\mathrm{PP})$ 管内, 在 $80^{\circ} \mathrm{C}$ 下保持 $1 \mathrm{~h}, \mathrm{GO}$ 在还原的同时也自组装成了 凝胶状纤维。溶液蒸发干燥之后, 石墨烯纤维极 度收缩, 直径减小了 $95 \%-97 \%$, 电导率达到 $8 \mathrm{~S} \cdot$ $\mathrm{cm}^{-1}$ 。在干法纺丝过程中, 液晶相分散液并不是必 需的, 分散杂乱的 $\mathrm{GO}$ 分散液以及低公称直径、低 
浓度的纺丝液也可以用来制备石墨烯纤维。改进 管状结构的形状以及在 $\mathrm{GO}$ 纺丝液中引入聚合物或 者金属纳米粒子掺杂, 可以制备出中空结构和石 墨烯复合纤维。

\subsubsection{3 干喷湿纺法制备石墨烯纤维}

干喷湿纺是合成石墨烯纤维的另一种重要的 方式。和湿法纺丝相比, 该方法也需要使用凝固 浴, 但是注射器喷头顶端与凝固浴未直接接触, 两者之间有一段空气间隙。采用干喷湿纺方式可 以制备出结构均匀, 截面环形的纤维 ${ }^{136}$ 。Xiang 等 ${ }^{137}$ 将 $\mathrm{GO}$ 分散在氯磺酸溶液中, $\mathrm{GO}$ 为液晶相, 喷头顶端与凝固浴之间的距离为 $12 \mathrm{~cm}$, 制备得到 了优异性能的石墨烯纤维。空气间隙的存在可以 减小喷头与凝固浴之间 $\mathrm{GO}$ 的速度梯度, 重力作用 可以改善纤维的取向性。研究发现, $12 \mathrm{~cm}$ 的空气 间隙得到的纤维直径比 $2 \mathrm{~cm}$ 的空气间隙时要小, 机械性能更好, 但是, 当空气间隙过长时纺丝效 果会变差。

\subsubsection{4 电泳法制备石墨烯纤维}

$\mathrm{GO}$ 分散液可视为胶体, 在电场的作用下, 带 电的 GO 片层会产生迁移运动出现电泳现象。Kim 等 ${ }^{138}$ 用电泳组装法制备出 GO 纤维。采用石墨针作 为正极, 将石墨针浸没在 GO 分散液中, 施加 1-2 $\mathrm{V}$ 的恒定电压, 在提拉石墨针过程中 $\mathrm{GO}$ 会在电极 的末端自组装成凝胶状纤维。干燥和退火之后, $\mathrm{rGO}$ 纤维具有光滑的表面。电泳法制备石墨烯纤维 效率较低, 不适合大规模生产。

\subsubsection{5 从石墨烯薄膜制备石墨烯纤维}

从石墨烯薄膜直接制备石墨烯纤维按石墨烯 的制备途径可分为两类。第一类是由 $\mathrm{GO}$ 薄膜卷绕 得到石墨烯纤维。Cruz-Silva 等 ${ }^{139}$ 在聚四氟乙烯薄 膜上刮涂 $\mathrm{GO}$ 溶液并蒸发溶剂得到连续的 GO 膜, 将 GO 薄膜裁剪成带状, 再卷绕成石墨烯纤维。第 二类是利用 $\mathrm{CVD}$ 生长出的石墨烯薄膜制备石墨烯 纤维。Zhu等 ${ }^{140,141}$ 先用 CVD 法在铜䈃上生长出连续 的石墨烯薄膜, 将铜箔基底刻蚀之后, 石墨烯薄 膜转移至有机溶剂中, 例如乙醇、丙酮以及乙酸 乙酯。这类有机溶剂的表现张力很小 $(22-25 \mathrm{mN}$. $\mathrm{m}^{-1}$ ), 石墨烯薄膜在这类溶剂中会皱缩, 当将石墨 烯薄膜拉出时, 由于三相界面张力(石墨烯、空气 以及有机溶剂)的作用, 会将卷曲的石墨烯薄膜挤 成纤维, 蒸发溶剂之后可以得到多孔石墨烯纤 维。这种方式得到的石墨烯纤维导电性较高, 可
以达到 10-200 $\mathrm{S} \cdot \mathrm{cm}^{-1}$, 但在刻蚀过程中石墨烯薄 膜易断裂, 且该方法不易于大面积制备。

\subsection{2 石墨烯纤维在柔性器件中的应用}

石墨烯纤维的柔性及优异的导电性、导热 性、机械和光电性能使其在柔性导体、能量存储 器件、驱动器、场发射器以及石墨烯弹簧等诸多 领域具有潜在的应用。

\subsubsection{1 石墨烯纤维柔性导体}

石墨烯纤维经过还原后具有很高的导电性, 可以满足作为电路连接导线的要求 ${ }^{142}$ 。 $\mathrm{Xu}$ 等 ${ }^{143}$ 使 用石墨烯纤维作为 LED 芯片的连接导线。石墨烯 纤维的柔性可以被编织成各种形态的织物或者加 入到纺织品中。Dong 等 ${ }^{144}$ 用石墨烯纤维编织成导 电织物, 使用PDMS 封装, 织物在机械变形时仍具 有较好的导电性。 $\mathrm{Xu}$ 等 ${ }^{111}$ 制备了银纳米线掺杂的 石墨烯纤维, 并将其转移至预拉伸的 PDMS 基底 上, 在 $50 \%$ 拉伸循环 50 次时可以保持电阻的稳定 性。这种轻质、高导电性的石墨烯纤维可以大批量 制备, 在轻质导电器件领域有广泛的应用前景。

\subsubsection{2 石墨烯纤维智能驱动器}

石墨烯纤维具有优异的驱动性能, 可以快速 的、可逆的响应环境刺激并产生形变。Wang 等 ${ }^{145}$ 制备了双层结构的石墨烯/聚吡咯(PPy)电压响应纤 维, 可以在外界电势的驱动下发生变形。 $\mathrm{Hu}$ 等 ${ }^{134}$ 制备了铂纳米粒子掺杂的中空石墨烯纤维, 在过 氧化氢溶液中, 因催化产生的气体可以驱动纤维 使其快速运动, 可被用作微制动器。 $\mathrm{Qu}$ 研究组 ${ }^{146}$ 用激光定位还原的方式制备了 $\mathrm{GO} / \mathrm{rGO}$ 不对称纤 维。这种纤维对湿度响应迅速, 可用作湿度驱动 的微制动器。

\subsubsection{3 石墨烯纤维柔性能源器件}

石墨烯纤维保持了石墨烯优异的电学、热学 和机械性质, 另外石墨烯纤维独特的一维结构和 优良的柔性使其在柔性能源器件领域具有很大的 应用潜力。一维纤维状的能源器件可以方便的集 成到衣物中, 从而在可穿戴设备、智能织物等领 域具有广泛的应用前景 ${ }^{147-150}$ 。Aboutalebi 等 ${ }^{151}$ 制备 了石墨烯纤维基超级电容器, 单电极比电容达到 了 $409 \mathrm{~F} \cdot \mathrm{g}^{-1}$, 石墨烯纤维的大比表面积 $\left(2210 \mathrm{~m}^{2}\right.$. $\left.\mathrm{g}^{-1}\right)$ 和高导电性 $\left(22.08 \mathrm{~S} \cdot \mathrm{cm}^{-1}\right)$ 是获得高电容的主要 原因。为了进一步提高电容性能, 一些研究组对 石墨烯纤维进行掺杂得到石墨烯纤维复合材料。 Cheng 等 ${ }^{152}$ 在 $\mathrm{rGO}$ 纤维外修饰了碳纳米管垂直阵 
列, 此纤维基超级电容器的电容达到了 $200.4 \mathrm{~F}$. $\mathrm{g}^{-1}$ 。 $\mathrm{Yu}$ 等 ${ }^{132}$ 制备了碳纳米管/氮掺杂石墨烯复合纤 维, 电导率达到 $102 \mathrm{~S} \cdot \mathrm{cm}^{-1}$ 。此纤维的电化学电容 达到了 $508 \mathrm{~F} \cdot \mathrm{g}^{-1}$, 是目前报道的碳材料基超级电 容器中比电容值最高的。

\subsubsection{4 其他方面的应用}

石墨烯纤维的高电导率、高电荷密度使其在 场发射器中有广泛的应用前景。Jang 等 ${ }^{138}$ 将石墨烯 纤维在 $800^{\circ} \mathrm{C}$ 下退火, 该纤维表现出优异的场发 射性能, 其驱动电压仅为 $0.7 \mathrm{~V} \cdot \mu \mathrm{m}^{-1}$, 电流密度达 到 $400 \mathrm{~A} \cdot \mathrm{cm}^{-2}$, 并且具有长时间发射稳定性。 Cruz-Sliva 等 ${ }^{139}$ 发现, 当石墨烯纤维在 $2000{ }^{\circ} \mathrm{C}$ 下 退火后, 驱动电压可以降至 $0.48 \mathrm{~V} \cdot \mu \mathrm{m}^{-1}$ 。Cheng 等 ${ }^{153}$ 将湿法纺丝得到的石墨烯纤维在玻璃棒上卷绕 得到石墨烯纤维弹簧, 经过高温退火之后, 纤维 可以保持弹簧形状，在 $480 \%$ 拉伸应变下仍具有优 异的回复性能。

石墨烯纤维是近年来发展出的新的碳基纤维 材料, 可由多种方法制备得到, 并且可以实现大 批量制备。石墨烯纤维具有优异的电学和力学性 能, 其电导率可以达到 $416 \mathrm{~S} \cdot \mathrm{cm}^{-1}$, 强度为 652 $\mathrm{MPa}$, 相比碳纳米管纤维和碳纤维还有很大的提升 空间。近期, 浙江大学高超研究组 ${ }^{154}$ 通过对石墨烯 纤维掺杂得到了电导率超过 $20000 \mathrm{~S} \cdot \mathrm{cm}^{-1}$ 的石墨烯 复合纤维。此外, 石墨烯纤维具有极好的柔性和 热导率, 在很多领域具有广泛的应用前景。

\section{4 碳纳米管/石墨烯基复合柔性导电材料}

如上所述, 碳纳米管和石墨烯已广泛用于柔 性导电材料的制备，使用单一的碳纳米管或者石 墨烯得到的导电材料的性能还有很大的提高空 间, 而将它们进行复合是改善材料性能的有效手 段之一, 复合途径包括 CVD 生长直接复合法和混 合溶液法。例如, Zhang 等 ${ }^{155}$ 将 CVD生长在铜簿 表面的石墨烯薄膜转移至沉积了金属催化剂薄膜 的基片上, 进一步用 CVD生长制备了石墨烯/碳纳 米管复合材料。 $\mathrm{Li}$ 等 ${ }^{156}$ 在铜箔上生长石墨烯薄膜之 后, 在表面覆盖上一层碳纳米管薄膜, 刻蚀铜基 底后, 即得到了碳纳米管/石墨烯复合薄膜。Shi 等 ${ }^{157}$ 先采用 FCCVD法在铜箔表面预沉积上一层碳 纳米管, 然后再生长石墨烯, 制备了碳纳米管穿 插在石墨烯中的网络结构, 这种复合结构薄膜在 刻蚀掉铜箔之后可以直接转移至柔性基底表面。
利用直接生长的方法得到的材料其结构和尺寸受 到的限制较多, 缺乏灵活性, 而溶液法过程简 单, 可大批量制备。例如, Tung 等 ${ }^{158}$ 在不添加表 面活性剂的情况下, 将碳纳米管和 $\mathrm{GO}$ 溶液混合, 得到了透光度为 $86 \%$, 电阻为 $240 \Omega \cdot \square^{-1}$ 的柔性导 电薄膜; 在混合溶液中加入水合肼, 将 $\mathrm{GO}$ 还原的 同时也保证了碳纳米管充分分散，将此薄膜用于 有机太阳能电池, 其太阳能转化效率达 $0.85 \%$ 。 Chen 等 ${ }^{159}$ 将碳纳米管和 $\mathrm{GO}$ 溶液在去离子水中分 散, 再加入对苯二酚和甲醛以及催化剂碳酸钠, 混合之后进行固化, 冷冻干燥以及 $1000^{\circ} \mathrm{C}$ 热解后 得到碳纳米管/ $\mathrm{GO}$ 气凝胶, 最后用 PDMS 预聚物在 真空辅助下填充得到了高导电可拉伸材料。Chen 等 ${ }^{160}$ 利用泡沫镍作为模板, 形成多孔的 PDMS (p-PDMS), 然后将 p-PDMS 浸没在 CNT/GO 溶液 中, 在 PDMS 表面形成导电层, 最后再用 $\mathrm{HI}$ 酸还 原。随着碳纳米管/ $\mathrm{GO}$ 溶液的浓度增大, 获得材料 的电导也随之增大, 渗透阈值非常小 $(2.5 \%)$, 得到 的三维可拉伸导体能在弯曲以及拉伸情况下多次 重复保证较好的导电性。

\section{5 结论与展望}

综上所述, 纳米碳材料因其优异的结构和性 能在柔性导电材料领域具有重要的应用价值。碳 纳米管是一种一维的碳材料, 其极佳的柔性和导 电性使其在柔性导电材料领域具有很大的应用潜 力。迄今为止, 基于碳纳米管阵列、碳纳米管薄 膜、碳纳米管纤维的柔性导电材料均有研究, 其 中碳纳米管薄膜作为柔性导电薄膜的研究最为成 熟, 碳纳米管薄膜和纤维的制备方法均包括直接 生长法和溶液法制备, 其中直接生长法得到碳纳 米管薄膜或者纤维缺陷含量低、结构完美、性能 优异, 但是该过程复杂、限制较多、成本较高, 因此工业化难度较大; 与之相比, 通过低成本的 碳纳米管粉体材料分散制备成溶液, 然后再制备 为薄膜或者纤维的方法更易于工业化生产, 在这 类方法中, 如何更好的设计过程, 在得到碳纳米 管分散性良好溶液的同时, 减少缺陷和杂质的引 入, 从而提高碳纳米管薄膜或者纤维的质量, 仍 然是研究的重点。碳纳米管薄膜或者纤维在柔性 场效应晶体管、集成电路、柔性触摸屏、储能以 及柔性传感等方面的应用已经进行了大量的探索 性研究, 但是, 要想真正的满足实际应用需要, 
还有很多工作要做。例如, 对于碳纳米管水平阵 列, 其密度和半导体纯度是其能否用于场效应器 件领域的关键, 然而目前还不能可控制备出高纯 度高密度的全半导体性碳纳米管水平阵列; 对于 从碳纳米管垂直阵列中直接抽丝得到的碳纳米管 薄膜, 虽然在触摸屏以及太阳能电池等领域已经 有了一定应用, 但是其透光率和导电性与 ITO 相比 还有待进一步提高, 成本也是制约其应用的因 素; 此外, 溶液法制备的碳纳米管薄膜和纤维则 存在缺陷含量大、强度较低、碳纳米管之间接触 电阻大因而产物电导率低等问题, 这有待于新工 艺的研究与开发。

与碳纳米管类似，石墨烯也具有良好的柔性 和导电性, 加之其制备方法更为灵活多样, 因此 在柔性导电材料领域也具有巨大的潜力。根据石 墨烯的来源, 主要分为两种途径, 一种是以天然 石墨为原料进行剥离而获得的石墨烯, 另一种是 CVD法制备的石墨烯。通过将天然石墨进行氧化 还原处理得到的氧化还原石墨烯, 其制备方法简 单、成本低、适于宏量制备, 但是含有大量缺 陷, 导电性能还有待进一步提高。而 CVD 法生长 的石墨烯, 需要采用卷对卷方式或者 PMMA 等聚 合物辅助转移, 很难制备大面积高质量的石墨烯 薄膜, 且因为操作过程中会导致石墨烯的掺杂和 破损, 从而降低石墨烯的导电性和电子迁移率, 目前其导电性能虽可满足其在柔性导电薄膜方面 的应用，但是仍有很大的提升空间。发展天然石 墨的更为温和绿色的解离方法、发展石墨烯的 CVD 低温生长技术和探索大面积无损转移工艺是 解决这些问题的可能途径。石墨烯纤维, 作为碳 基纤维家族的新成员，近几年也得到了广泛的关 注, 已发展出多种制备方法, 包括湿法纺丝、干 法纺丝、干喷释放、电泳法以及薄膜提拉成纤法 等。相比碳纳米管纤维和碳纤维, 石墨烯纤维的 电导率和机械性能较差, 但是通过对石墨烯纤维 掺杂可以较大程度提高电导率和力学性能。此 外, 石墨烯纤维优异的热导率和低廉的制备价格 使其在很多领域具有广阔的应用前景。

碳纳米管与石墨烯的结合以及它们与其他导 电纳米材料的结合可以制备出性能优异的纳米碳 材料复合材料，从而在保证柔性甚至透明性基础 上得到更高的电导率，是纳米碳材料基柔性导电 材料的一个重要发展方向。这类材料的进一步发
展也有赖于碳纳米管和石墨烯制备技术和分散技 术的发展。

纳米碳材料在柔性导电材料领域的应用已经 得到了长足的发展, 而且由于其原料来源多样, 制备过程灵活可控, 其中多种制备方法适于批量 化生产，因此具有巨大的发展潜力。我们相信， 随着相关技术的进一步成熟，纳米碳材料基柔性 导电材料会在未来有更大的突破，将伴随着智能 器件的发展而走进我们的日常生活, 不负社会寄 予这类材料的极大关注与期望。

\section{References}

(1) Kim, D. H.; Lu, N.; Ma, R.; Kim, Y. S.; Kim, R. H.; Wang, S.; Wu, J.; Won, S. M.; Tao, H.; Islam, A.; Yu, K. J.; Kim, T. I.; Chowdhury, R.; Ying, M.; Xu, L.; Li, M.; Chung, H. J.; Keum, H.; McCormick, M.; Liu, P.; Zhang, Y. W.; Omenetto, F. G.; Huang, Y.; Coleman, T.; Rogers, J. A. Science 2011, 333 (6044), 838. doi: 10.1126/science. 1206157

(2) Jeong, J. W.; Yeo, W. H.; Akhtar, A.; Norton, J. J. S.; Kwack, Y. J.; Li, S.; Jung, S. Y.; Su, Y.; Lee, W.; Xia, J.; Cheng, H.; Huang, Y.; Choi, W. S.; Bretl, T.; Rogers, J. A. Adv. Mater 2013, 25 (47), 6839. doi: 10.1002/adma.201301921

(3) Jeong, J. W.; Kim, M. K.; Cheng, H.; Yeo, W. H.; Huang, X.; Liu, Y.; Zhang, Y.; Huang, Y.; Rogers, J. A. Adv. Healthc. Mater. 2014, 3 (5), 642. doi: 10.1002/adhm.201300334

(4) Tao, H.; Brenckle, M. A.; Yang, M.; Zhang, J.; Liu, M.; Siebert, S. M.; Averitt, R. D.; Mannoor, M. S.; McAlpine, M. C.; Rogers, J. A.; Kaplan, D. L.; Omenetto, F. G. Adv. Mater. 2012, 24 (8), 1067. doi: 10.1002/adma.201103814

(5) Yeo, W. H.; Kim, Y. S.; Lee, J.; Ameen, A.; Shi, L.; Li, M.; Wang, S.; Ma, R.; Jin, S. H.; Kang, Z.; Huang, Y.; Rogers, J. A. Adv. Mater. 2013, 25 (20), 2773. doi: 10.1002/adma.201204426

(6) Webb, R. C.; Bonifas, A. P.; Behnaz, A.; Zhang, Y.; Yu, K. J.; Cheng, H.; Shi, M.; Bian, Z.; Liu, Z.; Kim, Y. S.; Yeo, W. H.; Park, J. S.; Song, J.; Li, Y.; Huang, Y.; Gorbach, A. M.; Rogers, J. A. Nat. Mater. 2013, 12 (10), 938. doi: 10.1038/nmat3755

(7) Huang, X.; Yeo, W. H.; Liu, Y.; Rogers, J. A. Biointerphases 2012, 7 (1), 52. doi: 10.1007/s13758-012-0052-8

(8) Huang, X.; Cheng, H.; Chen, K.; Zhang, Y.; Zhang, Y.; Liu, Y.; Zhu, C.; Ouyang, S. C.; Kong, G. W.; Yu, C.; Huang, Y.; Rogers, J. A. IEEE Trans. Biomed. Eng. 2013, 60 (10), 2848. doi: 10.1109/TBME.2013.2264879

(9) Huang, X.; Liu, Y.; Cheng, H.; Shin, W. J.; Fan, J. A.; Liu, Z.; Lu, C. J.; Kong, G. W.; Chen, K.; Patnaik, D.; Lee, S. H.; HageAli, S.; Huang, Y.; Rogers, J. A. Adv. Funct. Mater. 2014, 24 (25), 3846. doi: 10.1002/adfm.201303886

(10) Hattori, Y.; Falgout, L.; Lee, W.; Jung, S. Y.; Poon, E.; Lee, J. W.; Na, I.; Geisler, A.; Sadhwani, D.; Zhang, Y.; Su, Y.; Wang, X.; Liu, Z.; Xia, J.; Cheng, H.; Webb, R. C.; Bonifas, A. P.; 
Won, P.; Jeong, J. W.; Jang, K. I.; Song, Y. M.; Nardone, B.; Nodzenski, M.; Fan, J. A.; Huang, Y.; West, D. P.; Paller, A. S.; Alam, M.; Yeo, W. H.; Rogers, J. A. Adv. Healthc. Mater. 2014 3 (10), 1597. doi: 10.1002/adhm.201400073

(11) Huang, X.; Liu, Y.; Chen, K.; Shin, W. J.; Lu, C. J.; Kong, G. W.; Patnaik, D.; Lee, S. H.; Cortes, J. F.; Rogers, J. A. Small 2014, 10 (15), 3083. doi: 10.1002/smll.201400483

(12) Bian, Z.; Song, J.; Webb, R. C.; Bonifas, A. P.; Rogers, J. A.; Huang, Y. RSC Adv. 2014, 4 (11), 5694. doi: 10.1039/ c3ra45277h

(13) Bao, Z.; Lovinger, A. J. Chem. Mater. 1999, 11 (9), 2607. doi: $10.1021 / \mathrm{cm} 990290 \mathrm{~m}$

(14) Hammock, M. L.; Chortos, A.; Tee, B. C. K.; Tok, J. B. H.; Bao, Z. Adv. Mater. 2013, 25 (42), 5997. doi: 10.1002/ adma.201302240

(15) Heinze, J.; Frontana-Uribe, B. A.; Ludwigs, S. Chem. Mater. 2010, 110 (8), 4724. doi: $10.1021 / \mathrm{cr} 900226 \mathrm{k}$

(16) McQuade, D. T.; Pullen, A. E.; Swager, T. M. Chem. Mater. 2000, 100 (7), 2537. doi: 10.1021/cr9801014

(17) Arias, A. C.; MacKenzie, J. D.; McCulloch, I.; Rivnay, J.; Salleo, A. Chem. Mater. 2010, 110 (1), 3. doi: 10.1021/ cr900150b

(18) Park, S.; Vosguerichian, M.; Bao, Z. Nanoscale 2013, 5 (5), 1727. doi: $10.1039 / \mathrm{c} 3 \mathrm{nr} 33560 \mathrm{~g}$

(19) Hu, L.; Hecht, D. S.; Grüner, G. Chem. Mater. 2010, 110 (10), 5790. doi: $10.1021 / \mathrm{cr} 9002962$

(20) Mao, H. Y.; Laurent, S.; Chen, W.; Akhavan, O.; Imani, M.; Ashkarran, A. A.; Mahmoudi, M. Chem. Mater. 2013, 113 (5), 3407. doi: $10.1021 / \mathrm{cr} 300335 \mathrm{p}$

(21) Wu, W.; Wen, X.; Wang, Z. L. Science 2013, 340 (6135), 952. doi: $10.1126 /$ science. 1234855

(22) Takei, K.; Takahashi, T.; Ho, J. C.; Ko, H.; Gillies, A. G.; Leu, P. W.; Fearing, R. S.; Javey, A. Nat. Mater. 2010, 9 (10), 821. doi: $10.1038 /$ nmat2835

(23) Kim, K. S.; Zhao, Y.; Jang, H.; Lee, S. Y.; Kim, J. M.; Kim, K S.; Ahn, J. H.; Kim, P.; Choi, J. Y.; Hong, B. H. Nature 2009, 457 (7230), 706. doi: 10.1038/nature07719

(24) Zu, M.; Li, Q.; Wang, G.; Byun, J. H.; Chou, T. W. Adv. Funct. Mater. 2013, 23 (7), 789. doi: 10.1002/adfm. 201202174

(25) Lipomi, D. J.; Vosgueritchian, M.; Tee, B. C. K.; Hellstrom, S. L.; Lee, J. A.; Fox, C. H.; Bao, Z. Nat. Nanotechnol. 2011, 6 (12), 788. doi: 10.1038/nnano.2011.184

(26) Guo, T.; Nikolaev, P.; Rinzler, A. G.; Tomanek, D.; Colbert, D. T.; Smalley, R. E. J. Phys. Chem. 1995, 99 (27), 10694. doi: $10.1021 / \mathrm{j} 100027 \mathrm{a} 002$

(27) Xie, H.; Zhang, R.; Zhang, Y.; Yin, Z.; Jian, M.; Wei, F. Carbon 2016, 98, 157. doi: 10.1016/j.carbon.2015.11.001

(28) Huang, S.; Cai, X.; Liu, J. J. Am. Chem. Soc. 2003, 125 (19), 5636. doi: $10.1021 / \mathrm{ja} 034475 \mathrm{c}$

(29) Huang, S.; Cai, X.; Du, C.; Liu, J. J. Phys. Chem. B 2003, 107 (48), 13251. doi: 10.1021/jp0364708
(30) Huang, S.; Woodson, M.; Smalley, R.; Liu, J. Nano Lett. 2004, 4 (6), 1025. doi: 10.1021/n1049691d

(31) Joselevich, E.; Lieber, C. M. Nano Lett. 2002, 2 (10), 1137. doi: $10.1021 / \mathrm{nl} 025642 \mathrm{u}$

(32) Ismach, A.; Joselevich, E. Nano Lett. 2006, 6 (8), 1706. doi: $10.1021 / \mathrm{nl} 0610026$

(33) Ural, A.; Li, Y.; Dai, H. Appl. Phys. Lett. 2002, 81 (18), 3464. doi: $10.1063 / 1.1518773$

(34) Kang, S. J.; Kocabas, C.; Kim, H. S.; Cao, Q.; Meitl, M. A.; Khang, D. Y.; Rogers, J. A. Nano Lett. 2007, 7 (11), 3343. doi: $10.1021 / \mathrm{n} 1071596 \mathrm{~s}$

(35) Khang, D. Y.; Xiao, J.; Kocabas, C.; Maclaren, S.; Banks, T.; Jiang, H.; Huang, Y. Y.; Ja., R. Nano Lett. 2008, 8 (1), 124. doi: $10.1021 / \mathrm{nl} 072203 \mathrm{~s}$

(36) Kang, S. J.; Kocabas, C.; Ozel, T.; Shim, M.; Pimparkar, N.; Alam, M. A.; Rotkin, S. V.; Rogers, J. A. Nat. Nanotechnol. 2007, 2 (4), 230. doi:10.1038/nnano.2007.77

(37) Qing, C.; Hoon-Sik, K.; Ninad, P.; Kulkarni, J. P.; Congjun, W.; Moonsub, S.; Kaushik, R.; Alam, M. A.; Rogers, J. A. Nature 2008, 454 (454), 495. doi:10.1038/nature07110

(38) Tsai, T. Y.; Lee, C. Y.; Tai, N. H.; Tuan, W. H. Appl. Phys. Lett. 2009, 95 (1), 013103. doi: 10.1063/1.3174914

(39) Kyoon, S. M.; Jiyoung, O.; Marcio, L.; Kozlov, M. E.; Seon Jeong, K.; Baughman, R. H. Adv. Mater. 2010, 22 (24), 2663. doi: 10.1002/adma.200904270

(40) So, H. M.; Jin, W. S.; Kwon, J.; Yun, J.; Baik, S.; Chang, W. S. Mater. Res. Bull. 2013, 48 (12), 5036. doi: 10.1016/j. materresbull.2013.07.022

(41) Shin, U. H.; Jeong, D. W.; Kim, S. H.; Lee, H. W.; Kim, J. M. ACS Appl. Mater. Inter. 2014, 6 (15), 12909. doi: 10.1021/ am502851e

(42) Yamada, T.; Hayamizu, Y.; Yamamoto, Y.; Yomogida, Y.; IzadiNajafabadi, A.; Futaba, D. N.; Hata, K. Nat. Nanotechnol. 2011, 6 (5), 296. doi: 10.1038/nnano.2011.36

(43) Camponeschi, E.; Vance, R.; Al-Haik, M.; Garmestani, H.; Tannenbaum, R. Carbon 2007, 45 (10), 2037. doi: 10.1016/j. carbon.2007.05.024

(44) Dai, J.; Wang, Q.; Li, W.; Wei, Z.; Xu, G. Mater. Lett. 2007, 61 (1), 27. doi: 10.1016/j.matlet.2006.03.156

(45) Sekitani, T.; Noguchi, Y.; Hata, K.; Fukushima, T.; Aida, T.; Someya, T. Science 2008, 321 (5895), 1468. doi: 10.1126/ science. 1160309

(46) Sekitani, T.; Nakajima, H.; Maeda, H.; Fukushima, T.; Aida, T.; Hata, K.; Someya, T. Nat. Mater. 2009, 8 (6), 494 doi: $10.1038 /$ nmat2459

(47) Rogers, J. A.; Someya, T.; Huang, Y. Science 2010, 327 (5973), 1603. doi: $10.1126 /$ science. 1182383

(48) Lipomi, D. J.; Vosgueritchian, M.; Tee, B. C. K.; Hellstrom, S. L.; Lee, J. A.; Fox, C. H.; Bao, Z. Nat. Nanotechnol. 2011, 6 (12), 788. doi: 10.1038/nnano.2011.184

(49) Park, S.; Kim, H.; Vosgueritchian, M.; Cheon, S.; Kim, H.; 
Koo, J. H.; Kim, T. R.; Lee, S.; Schwartz, G.; Chang, H.; Bao, Z. Adv. Mater. 2014, 26 (43), 7324. doi: 10.1002/ adma.201402574

(50) Chun, K. Y.; Oh, Y.; Rho, J.; Ahn, J. H.; Kim, Y. J.; Choi, H. R.; Baik, S. Nat. Nanotechnol. 2010, 5 (12), 853. doi: 10.1038/ nnano.2010.232

(51) Ma, R.; Kwon, S.; Zheng, Q.; Kwon, H. Y.; Kim, J. I.; Choi, H. R.; Baik, S. Adv. Mater. 2012, 24 (25), 3344. doi: 10.1002/ adma.201201273

(52) Lee, J.; Woo, J. Y.; Kim, J. T.; Lee, B. Y.; Han, C. S. ACS Appl. Mater. Inter. 2014, 6 (14), 10974. doi: 10.1021/am502639n

(53) Zhang, M.; Fang, S.; Zakhidov, A. A.; Lee, S. B.; Aliev, A. E.; Williams, C. D.; Atkinson, K. R.; Baughman, R. H. Science 2005, 309 (5738), 1215. doi: 10.1126/science.1115311

(54) Zhang, X.; Jiang, K.; Feng, C.; Liu, P.; Zhang, L.; Kong, J.; Zhang, T.; Li, Q.; Fan, S. Adv. Mater. 2006, 18 (12), 1505. doi: 10.1002/adma.200502528

(55) Zhang, H. X.; Feng, C.; Zhai, Y. C.; Jiang, K. L.; Li, Q. Q.; Fan, S. S. Adv. Mater. 2009, 21 (22), 2299. doi: 10.1002/ adma. 200802290

(56) Jiang, K.; Wang, J.; Li, Q.; Liu, L.; Liu, C.; Fan, S. Adv. Mater. 2011, 23 (9), 1154. doi: 10.1002/adma.201003989

(57) Liu, K.; Sun, Y.; Liu, P.; Lin, X.; Fan, S.; Jiang, K. Adv. Funct. Mater. 2011, 21 (14), 2721. doi: 10.1002/adfm.201100306

(58) Feng, C.; Liu, K.; Wu, J. S.; Liu, L.; Cheng, J. S.; Zhang, Y.; Sun, Y.; Li, Q.; Fan, S.; Jiang, K. Adv. Funct. Mater. 2010, 20 (6), 885. doi: 10.1002/adfm.200901960

(59) Zhang, Y.; Sheehan, C. J.; Zhai, J.; Zou, G.; Luo, H.; Xiong, J.; Zhu, Y. T.; Jia, Q. X. Adv. Mater. 2010, 22 (28), 3027. doi: 10.1002/adma.200904426

(60) Xu, F.; Wang, X.; Zhu, Y.; Zhu, Y. Adv. Funct. Mater. 2012, 22 (6), 1279. doi: 10.1002/adfm.201102032

(61) Liu, Z. F.; Fang, S.; Moura, F. A.; Ding, J. N.; Jiang, N.; Di, J.; Zhang, M.; Lepro, X.; Galvao, D. S.; Haines, C. S.; Yuan, N. Y.; Yin, S. G.; Lee, D. W.; Wang, R.; Wang, H. Y.; Lv, W.; Dong, C.; Zhang, R. C.; Chen, M. J.; Yin, Q.; Chong, Y. T.; Zhang, R.; Wang, X.; Lima, M. D.; Ovalle-Robles, R.; Qian, D.; Lu, H.; Baughman, R. H. Science 2015, 349 (6246), 400. doi: $10.1126 /$ science.aaa7952

(62) Tawfick, S.; O'Brien, K.; Hart, A. J. Small 2009, 5 (21), 2467. doi: 10.1002/smll.200900741

(63) Cole, M.; Hiralal, P.; Ying, K.; Li, C.; Zhang, Y.; Teo, K.; Ferrari, A.; Milne, W. J. Nanomater. 2012, 272960. doi: $10.1155 / 2012 / 272960$

(64) In, J. B.; Lee, D.; Fornasiero, F.; Noy, A.; Grigoropoulos, C. P. ACS Nano 2012, 6 (9), 7858. doi: 10.1021/nn302192y

(65) Ma, W.; Song, L.; Yang, R.; Zhang, T.; Zhao, Y.; Sun, L.; Ren, Y.; Liu, D.; Liu, L.; Shen, J.; Zhang, Z.; Xiang, Y.; Zhou, W.; Xie, S. Nano Lett. 2007, 7 (8), 2307. doi: 10.1021/n1070915c

(66) Cai, L.; Li, J.; Luan, P.; Dong, H.; Zhao, D.; Zhang, Q.; Zhang, X.; Tu, M.; Zeng, Q.; Zhou, W.; Xie, S. Adv. Funct. Mater.
2012, 22 (24), 5238. doi: 10.1002/adfm.201201013

(67) Niu, Z.; Zhou, W.; Chen, J.; Feng, G.; Li, H.; Hu, Y.; Ma, W.; Dong, H.; Li, J.; Xie, S. Small 2013, 9 (4), 518. doi: 10.1002/ smll.201201587

(68) Vigolo, B.; Pénicaud, A.; Coulon, C.; Sauder, C.; Pailler, R.; Journet, C.; Bernier, P.; Poulin, P. Science 2000, 290 (5495), 1331. doi: 10.1126/science.290.5495.1331

(69) Macroscopic, N. Science 2004, 305, 1447. doi: 10.1126/ science. 1101398

(70) Vigolo, B.; Poulin, P.; Lucas, M.; Launois, P.; Bernier, P. Appl. Phys. Lett. 2002, 81 (7), 1210. doi: 10.1063/1.1497706

(71) Miaudet, P.; Badaire, S.; Maugey, M.; Derré, A.; Pichot, V.; Launois, P.; Poulin, P.; Zakri, C. Nano Lett. 2005, 5 (11), 2212. doi: $10.1021 / \mathrm{nl} 051419 \mathrm{w}$

(72) Kozlov, M. E.; Capps, R. C.; Sampson, W. M.; Ebron, V. H.; Ferraris, J. P.; Baughman, R. H. Adv. Mater. 2005, 17 (5), 614. doi: 10.1002/adma.200401130

(73) Steinmetz, J.; Glerup, M.; Paillet, M.; Bernier, P.; Holzinger, M. Carbon 2005, 43 (11), 2397. doi: 10.1016/j. carbon.2005.03.047

(74) Kozlov, M. E.; Capps, R. C.; Sampson, W. M.; Ebron, V. H.; Ferraris, J. P.; Baughman, R. H. Adv. Mater. 2005, 17 (5), 614. doi: 10.1002/adma.200401130

(75) Jiang, K.; Li, Q.; Fan, S. Nature 2002, 419 (6909), 801. doi: $10.1038 / 419801 \mathrm{a}$

(76) Zhang, M.; Atkinson, K. R.; Baughman, R. H. Science 2004, 306 (5700), 1358. doi: 10.1126/science. 1104276

(77) Xiefei, Z.; Qingwen, L.; Yi, T.; Yuan, L.; Coulter, J. Y.; Lianxi, Z.; Yonghao, Z.; Qianxi, J.; Peterson, D. E.; Yuntian, Z. Small 2007, 3 (2), 244. doi: 10.1002/smll.200600368

(78) Zhang, X.; Li, Q.; Holesinger, T. G.; Arendt, P. N.; Huang, J.; Kirven, P. D.; Clapp, T. G.; DePaula, R. F.; Liao, X.; Zhao, Y.; Zheng, L.; Peterson, D. E.; Zhu, Y. Adv. Mater. 2007, 19 (23), 4198. doi: 10.1002/adma.200700776

(79) Li, Y. L.; Kinloch, I. A.; Windle, A. H. Science 2004, 304 (5668), 276. doi: 10.1126/science.1094982

(80) Koziol, K.; Vilatela, J.; Moisala, A.; Motta, M.; Cunniff, P.; Sennett, M.; Windle, A. Science 2007, 318 (5858), 1892. doi: 10.1126/science. 1147635

(81) Zhong, X. H.; Li, Y. L.; Liu, Y. K.; Qiao, X. H.; Feng, Y.; Liang, J.; Jin, J.; Zhu, L.; Hou, F.; Li, J. Y. Adv. Mater. 2010, 22 (6), 692. doi: 10.1002/adma.200902943

(82) Aleman, B.; Reguero, V.; Mas, B.; Vilatela, J. J. ACS Nano 2015, 9 (7), 7392. doi: 10.1021/acsnano.5b02408

(83) Qu, G.; Cheng, J.; Li, X.; Yuan, D.; Chen, P.; Chen, X.; Wang, B.; Peng, H. Adv. Mater. 2016, 28 (19), 3646. doi: 10.1002/ adma.201600689

(84) Sun, G.; Zhang, X.; Lin, R.; Yang, J.; Zhang, H.; Chen, P. Angew. Chem. Int. Edit. 2015, 127 (15), 4734. doi: 10.1002/ ange.201411533

(85) Xu, R.; Wei, J.; Guo, F.; Cui, X.; Zhang, T.; Zhu, H.; Wang, K.; 
Wu, D. RSC Adv. 2015, 5 (28), 22015. doi: 10.1039/ C5RA01917F

(86) Hanaei, H.; Assadi, M. K.; Saidur, R. Renew. Sust. Energ. Rev. 2016, 59 620. doi: 10.1016/j.rser.2016.01.017

(87) Shang, Y.; Hua, C.; Xu, W.; Hu, X.; Wang, Y.; Zhou, Y.; Zhang, Y.; Li, X.; Cao, A. Nano lett. 2016, 16 (3), 1768. doi: 10.1021/ acs.nanolett.5b04773

(88) Qiu, L.; He, S.; Yang, J.; Deng, J.; Peng, H. Small 2016, 12 (18), 2419. doi: 10.1002/smll.201600326

(89) Zhang, Y.; Zhao, Y.; Ren, J.; Weng, W.; Peng, H. Adv. Mater. 2016, 28, 4524. doi: 10.1002/adma.201503891

(90) Novoselov, K. S.; Geim, A. K.; Morozov, S. V.; Jiang, D.; Zhang, Y.; Dubonos, S. V.; Grigorieva, I. V.; Firsov, A. A. Science 2004, 306 (5696), 666. doi: 10.1126/science.1102896

(91) Rogala, M.; Wlasny, I.; Dabrowski, P.; Kowalczyk, P. J.; Busiakiewicz, A.; Kozlowski, W.; Lipinska, L.; Jagiello, J.; Aksienionek, M.; Strupinski, W.; Krajewska, A.; Sieradzki, Z.; Krucinska, I.; Puchalski, M.; Skrzetuska, E.; Klusek, Z. Appl. Phys. Lett. 2015, 106 (4), 041901. doi: 10.1063/1.4906593

(92) Jiang, W. S.; Liu, Z. B.; Xin, W.; Chen, X. D.; Tian, J. G. Nanotechnology 2016, 27 (9), 095301. doi: 10.1088/09574484/27/9/095301

(93) Eda, G.; Fanchini, G.; Chhowalla, M. Nat. Nanotechnol. 2008, 3 (5), 270. doi: 10.1038/nnano.2008.83

(94) Kim, S. H.; Yu, Y.; Li, Y. Z.; Xu, T.; Zhi, J. F. J. Mater. Chem 2012, 22 (35), 18306. doi: 10.1039/C2JM31048A

(95) Becerril, H. A.; Mao, J.; Liu, Z.; Stoltenberg, R. M.; Bao, Z.; Chen, Y. ACS Nano 2008, 2 (3), 463. doi: 10.1021/nn700375n

(96) Robinson, J. T.; Zalalutdinov, M.; Baldwin, J. W.; Snow, E. S.; Wei, Z.; Sheehan, P.; Houston, B. H. Nano Lett. 2008, 8 (10), 3441. doi: 10.1021/n18023092

(97) Chang, H.; Wang, G.; Yang, A.; Tao, X.; Liu, X.; Shen, Y.; Zheng, Z. Adv. Funct. Mater. 2010, 20 (17), 2893. doi: 10.1021/ n18023092

(98) Zheng, Q.; Ip, W. H.; Lin, X.; Yousefi, N.; Yeung, K. K.; Li, Z.; Kim, J. K. ACS Nano 2011, 5 (7), 6039. doi: 10.1002/ adfm. 201000900

(99) Pei, S.; Zhao, J.; Du, J.; Ren, W.; Cheng, H. M. Carbon 2010, 48 (15), 4466. doi: 10.1021/nn2018683

(100) Chen, C.; Yang, Q. H.; Yang, Y.; Lv, W.; Wen, Y.; Hou, P. X.; Wang, M.; Cheng, H. M. Adv. Mater. 2009, 21 (29), 3007. doi: 10.1002/adma.200803726

(101) Kim, J.; Cote, L. J.; Kim, F.; Huang, J. J. Am. Chem. Soc. 2010, 132 (1), 260. doi: 10.1016/j.carbon.2010.08.006

(102) Li, X.; Zhang, G.; Bai, X.; Sun, X.; Wang, X.; Wang, E.; Dai, H. Nat. Nanotechnol. 2008, 3 (9), 538. doi: 10.1038/ nnano.2008.210

(103) Wu, Z. S.; Pei, S.; Ren, W.; Tang, D.; Gao, L.; Liu, B.; Li, F.; Liu, C.; Cheng, H. M. Adv. Mater. 2009, 21 (17), 1756. doi: 10.1002/adma.200802560

(104) Moon, I. K.; Lee, J.; Ruoff, R. S.; Lee, H. Nat. Commun. 2010,
1,73. doi: 10.1038/ncomms1067

(105) Wang, J.; Liang, M.; Fang, Y.; Qiu, T.; Zhang, J.; Zhi, L. Adv Mater. 2012, 24 (21), 2874. doi: 10.1002/adma.201200055

(106) Lv, X.; Huang, Y.; Liu, Z.; Tian, J.; Wang, Y.; Ma, Y.; Liang, J.; Fu, S.; Wan, X.; Chen, Y. Small 2009, 5 (14), 1682. doi: $10.1002 /$ smll.200900044

(107) Tien, H. W.; Hsiao, S. T.; Liao, W. H.; Yu, Y. H.; Lin, F. C.; Wang, Y. S.; Li, S. M.; Ma, C. C. M. Carbon 2013, 58, 198. doi: 10.1016/j.carbon.2013.02.051

(108) Liu, Y.; Chang, Q.; Huang, L. J. Mater. Chem. C 2013, 1 (17), 2970. doi: $10.1039 / \mathrm{C} 3 \mathrm{TC} 30178 \mathrm{H}$

(109) Jeong, C.; Nair, P.; Khan, M.; Lundstrom, M.; Alam, M. A. Nano Lett. 2011, 11 (11), 5020. doi: 10.1021/n1203041n

(110) Choi, H. O.; Kim, D. W.; Kim, S. J.; Yang, S. B.; Jung, H. T. Adv. Mater. 2014, 26 (26), 4575. doi: 10.1002/adma.201306234

(111) Xu, Z.; Liu, Z.; Sun, H.; Gao, C. Adv. Mater. 2013, 25 (23), 3249. doi: 10.1002/adma.201300774

(112) Zhu, Y.; Sun, Z.; Yan, Z.; Jin, Z.; Tour, J. M. ACS Nano 2011, 5 (8), 6472. doi: 10.1021/nn201696g

(113) Xia, K.; Jian, M.; Zhang, W.; Zhang, Y. Adv. Mater. Inter. 2016, 3 (6), 1500674. doi: 10.1002/admi.201500674

(114) Li, X.; Zhu, Y.; Cai, W.; Borysiak, M.; Han, B.; Chen, D.; Piner, R. D.; Colombo, L.; Ruoff, R. S. Nano Lett. 2009, 9 (12), 4359. doi: 10.1021/n1902623y

(115) Bae, S.; Kim, H.; Lee, Y.; Xu, X.; Park, J. S.; Zheng, Y.; Balakrishnan, J.; Lei, T.; Kim, H. R.; Song, Y. I.; Kim, Y. J.; Kim, K. S.; Ozyilmaz, B.; Ahn, J. H.; Hong, B. H.; Iijima, S. Nat. Nanotechnol. 2010, 5 (8), 574. doi: 10.1038/ nnano.2010.132

(116) Won, S.; Hwangbo, Y.; Lee, S. K.; Kim, K. S.; Kim, K. S.; Lee, S. M.; Lee, H. J.; Ahn, J. H.; Kim, J. H.; Lee, S. B. Nanoscale 2014, 6 (11), 6057. doi: 10.1039/C4NR00265B

(117) Chen, T.; Xue, Y.; Roy, A. K.; Dai, L. ACS Nano 2014, 8 (1), 1039. doi: 10.1021/nn405939w.

(118) Xu, Z.; Gao, C. Mater. Today 2015, 18 (9), 480. doi: 10.1016/j. mattod.2015.06.009

(119) Chen, L. L.; Liu, Y.; Zhao, Y.; Chen, N.; Qu, L. T. Nanotechnology 2016, 27 (3), 032001. doi: 10.1088/0957-4484/ 27/3/032001

(120) Meng, F.; Lu, W.; Li, Q.; Byun, J. H.; Oh, Y.; Chou, T. W. Adv. Mater. 2015, 27 (35), 5113. doi: 10.1002/adma.201501126

(121) Jalili, R.; Aboutalebi, S. H.; Esrafilzadeh, D.; Shepherd, R. L.; Chen, J.; Aminorroaya-Yamini, S.; Konstantinov, K.; Minett, A. I.; Razal, J. M.; Wallace, G. G. Adv. Funct. Mater. 2013, 23 (43), 5345. doi: 10.1002/adfm.201300765

(122) Xu, Z.; Sun, H.; Zhao, X.; Gao, C. Adv. Mater. 2013, 25 (2), 188. doi: $10.1002 /$ adma.201203448

(123) Liu, Z.; Xu, Z.; Hu, X.; Gao, C. Macromolecules 2013, 46 (17), 6931. doi: 10.1021/ma400681v

(124) Hu, X.; Xu, Z.; Liu, Z.; Gao, C. Sci. Rep. 2013, 3, 2374. doi: $10.1038 /$ srep 02374 
(125) Zhao, X.; Xu, Z.; Zheng, B.; Gao, C. Sci. Rep. 2013, 3, 3164. doi: $10.1038 /$ srep03164

(126) Xiang, C.; Young, C. C.; Wang, X.; Yan, Z.; Hwang, C. C.; Cerioti, G.; Lin, J.; Kono, J.; Pasquali, M.; Tour, J. M. $A d v$. Mater. 2013, 25 (33), 4592. doi: 10.1002/adma.201301065

(127) Cao, J.; Zhang, Y.; Men, C.; Sun, Y.; Wang, Z.; Zhang, X.; Li, Q. ACS Nano 2014, 8 (5), 4325. doi: 10.1021/nn4059488

(128) Meng, F.; Li, R.; Li, Q.; Lu, W.; Chou, T. W. Carbon 2014, 72, 250. doi: 10.1016/j.carbon.2014.01.073

(129) Kim, Y. S.; Kang, J. H.; Kim, T.; Jung, Y.; Lee, K.; Oh, J. Y.; Park, J.; Park, C. R. Chem. Mater. 2014, 26 (19), 5549. doi: $10.1021 / \mathrm{cm} 502614 \mathrm{w}$

(130) Kou, L.; Huang, T.; Zheng, B.; Han, Y.; Zhao, X.; Gopalsamy, K.; Sun, H.; Gao, C. Nat. Commun. 2014, 5, 3754. doi: $10.1038 /$ ncomms 4754

(131) Zhao, Y.; Jiang, C.; Hu, C.; Dong, Z.; Xue, J.; Meng, Y.; Zheng, N.; Chen, P.; Qu, L. ACS Nano 2013, 7 (3), 2406. doi: $10.1021 / \mathrm{nn} 305674 \mathrm{a}$

(132) Yu, D.; Goh, K.; Wang, H.; Wei, L.; Jiang, W.; Zhang, Q.; Dai, L.; Chen, Y. Nat. Nanotechnol. 2014, 9 (7), 555. doi: 10.1038/ nnano. 2014.93

(133) Acik, M.; Lee, G.; Mattevi, C.; Pirkle, A.; Wallace, R. M.; Chhowalla, M.; Cho, K.; Chabal, Y. J. Phys. Chem. C 2011, 115 (40), 19761. doi: 10.1021/jp2052618

(134) Hu, C.; Zhao, Y.; Cheng, H.; Wang, Y.; Dong, Z.; Jiang, C.; Zhai, X.; Jiang, L.; Qu, L. Nano Lett. 2012, 12 (11), 5879. doi: $10.1021 / \mathrm{nl} 303243 \mathrm{~h}$

(135) Li, J.; Li, J.; Li, L.; Yu, M.; Ma, H.; Zhang, B. J. Mater. Chem. A 2014, 2 (18), 6359. doi: 10.1039/C4TA00431K

(136) Zhang, J.; Zhang, Y.; Zhang, D.; Zhao, J. J. Appl. Polym. Sci. 2012, 125 (S1), E58. doi: 10.1002/app.36317

(137) Xiang, C.; Behabtu, N.; Liu, Y.; Chae, H. G.; Young, C. C.; Genorio, B.; Tsentalovich, D. E.; Zhang, C.; Kosynkin, D. V.; Lomeda, J. R.; Hwang, C. C.; Kumar, S.; Pasquali, M.; Tour, J. M. ACS Nano 2013, 7 (2), 1628. doi: 10.1021/nn305506s

(138) Jang, E. Y.; Carretero-Gonzalez, J.; Choi, A.; Kim, W. J.; Kozlov, M. E.; Kim, T.; Kang, T. J.; Baek, S. J.; Kim, D. W.; Park, Y. W.; Baughman, R. H.; Kim, Y. H. Nanotechnology 2012, 23 (23), 235601. doi: 10.1088/0957-4484/23/23/235601

(139) Cruz-Silva, R.; Morelos-Gomez, A.; Kim, H. I.; Jang, H. K.; Tristan, F.; Vega-Diaz, S.; Rajukumar, L. P.; Elías, A. L.; PereaLopez, N.; Suhr, J.; Endo, M.; Terrones, M. ACS Nano 2014, 8 (6), 5959. doi: 10.1021/nn501098d

(140) Li, X.; Zhao, T.; Wang, K.; Yang, Y.; Wei, J.; Kang, F.; Wu, D.; Zhu, H. Langmuir 2011, 27 (19), 12164. doi: 10.1021/ la202380g

(141) Li, X.; Zhao, T.; Chen, Q.; Li, P.; Wang, K.; Zhong, M.; Wei, J.; Wu, D.; Wei, B.; Zhu, H. Phys. Chem. Chem. Phys 2013, 15 (41), 17752. doi: 10.1039/C3CP52908H

(142) Fang, B.; Peng, L.; Xu, Z.; Gao, C. ACS Nano 2015, 9 (5),
5214. doi: 10.1021/acsnano.5b00616

(143) Xu, Z.; Zhang, Y.; Li, P.; Gao, C. ACS Nano 2012, 6 (8), 7103. doi: $10.1021 / \mathrm{nn} 3021772$

(144) Dong, Z.; Jiang, C.; Cheng, H.; Zhao, Y.; Shi, G.; Jiang, L.; Qu, L. Adv. Mater. 2012, 24 (14), 1856. doi: 10.1002/ adma. 201200170

(145) Wang, Y.; Bian, K.; Hu, C.; Zhang, Z.; Chen, N.; Zhang, H.; Qu, L. Electrochem. Commun. 2013, 35, 49. doi: 10.1016/j. elecom.2013.07.044

(146) Cheng, H.; Liu, J.; Zhao, Y.; Hu, C.; Zhang, Z.; Chen, N.; Jiang, L.; Qu, L. Angew. Chem. Int. Edit. 2013, 52 (40), 10482. doi: 10.1002/anie.201304358

(147) Chen, S. H.; Ma, W. J.; Cheng, Y. H.; Weng, Z.; Sun, B.; Wang, L.; Chen, W. P.; Li, F.; Zhu, M. F.; Cheng, H. M. Nano Energy 2015, 15, 642. doi: 10.1016/j.nanoen.2015.05.004

(148) Ma, Y. W.; Li, P.; Sedloff, J. W.; Zhang, X.; Zhang, H. B.; Liu, J. ACS Nano 2015, 9 (2), 1352. doi: 10.1021/nn505412v

(149) Chen, S. H.; Ma, W. J.; Xiang, H. X.; Cheng, Y. H.; Yang, S. Y.; Weng, W.; Zhu, M. F. J. Power Sources 2016, 319, 271. doi: 10.1016/j.jpowsour.2016.04.030

(150) Jia, X. L.; Lu, Y. F.; Wei, F. Nano Res. 2016, 9 (1), 230. doi: 10.1007/s12274-016-1001-5

(151) Aboutalebi, S. H.; Jalili, R.; Esrafilzadeh, D.; Salari, M.; Gholamvand, Z.; Yamini, S. A.; Konstantinov, K.; Shepherd, R. L.; Chen, J.; Moulton, S. E.; Innis, P. C.; Minett, A. I.; Razal, J. M.; Wallace, G. G. ACS Nano 2014, 8 (3), 2456. doi: $10.1021 / \mathrm{nn} 406026 \mathrm{z}$

(152) Cheng, H.; Dong, Z.; Hu, C.; Zhao, Y.; Hu, Y.; Qu, L.; Chen, N.; Dai, L. Nanoscale 2013, 5 (8), 3428. doi: 10.1039/ c3nr00320e

(153) Cheng, H.; Liang, Y.; Zhao, F.; Hu, Y.; Dong, Z.; Jiang, L.; Qu, L. Nanoscale 2014, 6 (19), 11052. doi: 10.1039/c4nr03409k

(154) Liu, Y.; Xu, Z.; Zhan, J.; Li, P.; Gao, C. Adv. Mater. 2016, doi: 10.1002/adma.201602444

(155) Zhang, W.; Xie, H.; Zhang, R.; Jian, M.; Wang, C.; Zheng, Q.; Wei, F.; Zhang, Y. Carbon 2015, 86, 358. doi: 10.1016/j. carbon.2015.01.051

(156) Li, C.; Li, Z.; Zhu, H.; Wang, K.; Wei, J.; Li, X.; Sun, P.; Zhang, H.; Wu, D. J. Phys. Chem. C 2010, 114 (33), 14008. doi: $10.1021 /$ jp 1041487

(157) Shi, E.; Li, H.; Yang, L.; Hou, J.; Li, Y.; Li, L.; Cao, A.; Fang, Y. Adv. Mater. 2015, 27 (4), 682. doi: 10.1002/adma.201403722

(158) Tung, V. C.; Chen, L. M.; Allen, M. J.; Wassei, J. K.; Nelson, K.; Kaner, R. B.; Yang, Y. Nano Lett. 2009, 9 (5), 1949. doi: $10.1021 / \mathrm{n} 19001525$

(159) Chen, M.; Tao, T.; Zhang, L.; Gao, W.; Li, C. Chem. Commun. 2013, 49 (16), 1612. doi: 10.1039/c2cc38290c

(160) Chen, M.; Zhang, L.; Duan, S.; Jing, S.; Jiang, H.; Li, C. $A d v$. Funct. Mater. 2014, 24 (47), 7548. doi: 10.1002/ adfm.201401886 